\title{
BDNF gene delivery mediated by neuron- targeted nanoparticles is neuroprotective in peripheral nerve injury
}

Cátia D.F. Lopes a,b,c, Nádia P. Gonçalves a,d,1, Carla P. Gomes a,b,e, Maria J. Saraiva a,d, Ana P. Pêgo a,b,e,f,

a i3S - Instituto de Investigaçao e Inovação em Saúde, Universidade do Porto, Rua Alfredo Allen, 208, 4200-135 Porto, Portugal

b INEB - Instituto de Engenharia Biomédica, Universidade do Porto, Rua Alfredo Allen, 208, 4200 -

135 Porto, Portugal

c FMUP - Faculdade de Medicina da Universidade do Porto, Alameda Prof. Hernâni Monteiro, 4200319 Porto, Portugal

d IBMC - Instituto de Biologia Molecular e Celular, Universidade do Porto, Rua Alfredo Allen, 208, 4200-135 Porto, Portugal

e FEUP - Faculdade de Engenharia, Universidade do Porto, Porto, R. Dr. Roberto Frias s/n, 4200465 Porto, Portugal

f ICBAS - Instituto de Ciências Biomédicas Abel Salazar, Universidade do Porto, R. Jorge de Viterbo Ferreira 228, Porto, Portugal

* Corresponding author. i3S, Rua Alfredo Allen, 208, 4200-135 Porto, Portugal.

E-mail address: apego@ineb.up.pt (A.P. Pego).

1 Presently at Danish Research Institute of Translational Neuroscience DANDRITE, Nordic EMBL Partnership, Department of Biomedicine, Aarhus University, Denmark.

Originally published in Biomaterials 121, 83-96, December 24, 2016.

DOI: 10.1016/j.biomaterials.2016.12.025

Keywords: Neuron-targeted nanoparticles; Gene therapy; Neuropathies; Neuroprotection; Trimethyl chitosan; Brain-derived neurotrophic factor

Abstract

Neuron-targeted gene delivery is a promising strategy to treat peripheral neuropathies. Here we propose the use of polymeric nanoparticles based on thiolated trimethyl chitosan (TMCSH) to mediate targeted gene delivery to peripheral neurons upon a peripheral and minimally invasive intramuscular administration. Nanoparticles were grafted with the non-toxic carboxylic fragment of the tetanus neurotoxin (HC) to allow neuron targeting and were explored to deliver a plasmid DNA encoding for the brain-derived neurotrophic factor (BDNF) in a peripheral nerve injury model. The TMCSH-HC/BDNF nanoparticle treatment promoted the release and significant expression of BDNF in neural tissues, which resulted in an enhanced functional recovery after injury as compared to control treatments (vehicle and non-targeted nanoparticles), associated with an improvement in 
key pro-regenerative events, namely, the increased expression of neurofilament and growth-associated protein GAP-43 in the injured nerves. Moreover, the targeted nanoparticle treatment was correlated with a significantly higher density of myelinated axons in the distal stump of injured nerves, as well as with preservation of unmyelinated axon density as compared with controls and a protective role in injury-denervated muscles, preventing them from denervation. These results highlight the potential of TMCSH-HC nanoparticles as non-viral gene carriers to deliver therapeutic genes into the peripheral neurons and thus, pave the way for their use as an effective therapeutic intervention for peripheral neuropathies.

\section{Introduction}

Treatment of peripheral neuropathies has always been a clinical challenge. Peripheral neuropathies may take weeks to months to develop after the exposure of the neural tissue to the insulting agents and often these cause irreversible damage to the affected nerves. Presently, there is a single clinical therapeutic strategy available, consisting into the identification of the peripheral neuropathy etiology and, if possible, treatment of the underlying cause and/or management of the associated symptoms [1]. There is no treatment addressing the damaged nerves, which makes vital the development of effective regenerative strategies.

Neurotrophic factors have been extensively studied as a restorative treatment option for peripheral neuropathies due to their role in neuronal survival, growth and synaptic plasticity [2]. The promising results of several neurotrophic factors in pre-clinical tests [2] are consensual and have prompted their evaluation in clinical trials (in which the recombinant proteins have been directly administered to patients) but the outcomes have failed to demonstrate a significant beneficial effect of these agents $[3,4]$. One justification for this failure can be attributed to the employed administration strategy that was either systemic or subcutaneous. The blood-nerve barrier, the interface that isolates the endoneurium from the intravascular component, is relatively impermeable to molecules of larger sizes (such as neurotrophic factors) and, thus, prevents the exit of such factors from circulation to reach the targeted cells $[5,6]$. Moreover, due to their short systemic half-life and pleiotropic actions, the direct administration of neurotrophic factors can cause systemic, as well as peripheral adverse effects that overrule the desired protective action [7]. Thus, novel delivery tools and application forms are being explored to attain the efficient local delivery of neurotrophic factors while limiting their action to the target area to prevent unwanted adverse side-effects [8].

Gene therapy has been considered as one of the promising strategies to treat both inherited and acquired diseases. The principle of gene therapy relies on the use of nucleic acids as a pro-drug that can lead to the modulation of the expression of therapeutic proteins within cells. In this sense, it has been proposed as an approach to promote the local expression of neurotrophic factors and restore the functional properties of the damaged nerves in numerous neuropathic conditions [9].

Recently, extensive research is being conducted towards the design of safe and effective nonviral vectors that could achieve comparable levels of gene expression and specificity attained by viral vectors, while providing greater flexibility in terms of cargo size, immunological compatibility and reduced safety concerns [9]. Among these, chitosan-based carriers have gained special attention due to chitosan ability to condense nucleic acids and protect them from 
endonuclease degradation, its biocompatibility and biodegradability [10]. In particular, the use of quaternized chitosan derivatives overcomes one of the major caveats of this polycation, the low $\mathrm{pKa}$ that leads to its low solubility at physiologic $\mathrm{pH}$. The presence of permanent positively charged groups in the chitosan backbone enables the electrostatic interactions between the polymer and the negatively charged nucleic acids to occur even under physiological conditions, improving the stability of the resulting polyplexes, an important feature for their applicability in vivo [11].

Here we propose the use of a polymeric nanoparticle based on trimethyl chitosan (TMC) to vectorize therapeutic genes to peripheral neurons in vivo and in a targeted fashion, after a peripheral and minimally invasive intramuscular administration. The explored nanoparticletargeting moiety was the non-toxic carboxylic terminal fragment from the tetanus neurotoxin (HC), which we have previously shown to mediate nanoparticle retrograde transport after a peripheral administration in the proximity of neuron terminals [12,13]. The performance of this neuron-targeted TMC-based nanoformulation to deliver therapeutic genes to peripheral neurons and rescue them from degeneration was assessed in vivo, in a peripheral nerve crush injury animal model, using as therapeutic transgene a plasmid DNA encoding for the brainderived neurotrophic factor (BDNF). The rationale for using BDNF is based on its reported protective role in neuronal survival after injury. BDNF has been advocated as a potential candidate to promote nerve protection and/or regeneration for the following well-established reasons. After peripheral nerve injury BDNF mRNA is up-regulated in dorsal root ganglia (DRG), Schwann cells and target muscles fibers [14e16]. At protein level, BDNF expression is also increased in the DRG and in the spinal dorsal horn after an injury $[15,17,18]$. It has also been found that the high affinity tyrosine kinase receptor B (trkB), one of the major BDNF cell receptors, is overexpressed in regenerating sensory and motor neurons $[19,20]$. Moreover, the deprivation of endogenous BDNF results in an impairment of regeneration and remyelination of peripheral nerves [14]. These observations have pointed to the key role of BDNF in nerve regeneration mediated by the trkB receptors present in motor and a subset of sensory neurons, making this neurotrophic factor of critical relevance to be applied in the treatment of motor and sensory dysfunctions present in peripheral neuropathies [14]. Previous studies showed that BDNF gene therapy mediated by viral vectors corroborate the crucial role of BDNF in conditions such as blindness [21e23], deafness [24,25], sciatic nerve [26] and adult motor neuron injuries [27]. Despite the promising results obtained so far, the in vivo application of viral vectors has demonstrated problems of toxicity, immunogenicity and inflammatory potential. In this study, using a peripheral nerve crush injury model, in which nerve degeneration and regeneration occur in a well-established cascade of events, we were able to establish the effect of the proposed TMCSH-HC/BDNF nanoparticles in protecting peripheral nerves from degeneration and enhancing the speed of nerve regeneration.

\section{Materials and Methods}

\subsection{TMC purification and modification}

TMC derived from ultrapure chitosan produced from Agaricus bisporus mushrooms, under cGMP standards, (40 kDa, lot VIHA0013-157, KitoZyme, Belgium) was purified by filtration and dialysis prior to use, as previously described [28]. In brief, TMC was diluted in $5 \mathrm{mM} \mathrm{HCl}$ solution at a final concentration of $0.5 \%(\mathrm{w} / \mathrm{v})$, filtered through a Buchner funnel and purified by dialysis using a $3.5 \mathrm{kDa}$ molecular weight cut-off membrane (Spectrum Labs, CA, USA) for 3 days against deionized water and collected after freezedrying. The degree of acetylation (DA) and 
quaternization (DQ) of the purified TMC were determined by ${ }^{1} \mathrm{H}-$ nuclear magnetic resonance spectroscopy ( ${ }^{1} \mathrm{H}-\mathrm{NMR}$; see Fig. S1 in Supplementary Information for a characteristic TMC ${ }^{1} \mathrm{H}-$ NMR spectrum) and found to be $15.7 \pm 0.9 \%$ and $30.1 \pm 4.6 \%$, respectively. Endotoxin levels of the purified polymer extracts were assessed using the Limulus Amebocyte Lysate Assay (QCL1000, Cambrex, NJ, USA), following the manufacturer instructions. Endotoxin levels were found to be $<0.1 \mathrm{EU} / \mathrm{ml}$ (an EU corresponding to a unit of measurement for endotoxin activity), respecting the US Department of Health and Human Services guidelines for implantable devices [29]. After freeze-dried, TMC was dissolved in $20 \mathrm{mM}$ HEPES (Sigma-Aldrich Co, MO, USA) buffered saline solution containing $5 \%(\mathrm{w} / \mathrm{v})$ glucose $(\mathrm{pH} 7.4)$ at a $0.25 \%(\mathrm{w} / \mathrm{v})$ final concentration. Purified TMC was then thiolated (for reaction scheme - see Fig. S2 in Supplementary Information) by the tethering of thiol groups from 2-iminothiolane on the primary amino groups of the polymer, as described elsewhere [12]. Briefly, TMC was dissolved in $20 \mathrm{mM} \mathrm{HEPES}$ buffer at $5 \mathrm{mg} / \mathrm{ml}$ and 2-iminothiolane $\mathrm{HCl}$ (Sigma-Aldrich Co) was added to

the polymer solution, in order to attain a theoretical modification of $10 \%$ of the polymer primary amine groups. Afterwards, the pH of the solution was adjusted to 8 with $\mathrm{NaOH}$. After $6 \mathrm{~h}$ of incubation at room temperature (RT), under an inert atmosphere saturated with argon and with continuous stirring, the resulting thiolated TMC (TMCSH) was dialyzed for 3 days against $5 \mathrm{mM}$ $\mathrm{HCl}$ at $4 \stackrel{\circ}{\circ}$. Thereafter samples were frozen at $-80{ }^{\circ} \mathrm{C}$ and lyophilized for 3 days. The resulting powders were stored at $-20 \stackrel{\circ}{ } \mathrm{C}$ until further use.

The degree of polymer thiolation was quantified with 5,5-dithio-bis-(2-nitrobenzoic acid) (DTNB, Ellman's reagent). The amount of free thiol groups grafted to the polymer was determined based on a previously described protocol with some modifications [30] and found to be $149.1 \pm 15.8 \mu \mathrm{mol} / \mathrm{g}$, corresponding to $4.7 \pm 0.5 \%$ substitution of TMC primary amines by thiol groups. Briefly, DTNB was dissolved in $0.1 \mathrm{M}$ sodium phosphate buffer, $\mathrm{pH} 8$ at a final concentration of $100 \mu \mathrm{g} / \mathrm{ml}$. The polymer samples were dissolved in the same solution at a final concentration of $2.5 \mathrm{mg} / \mathrm{ml}$. Then, $20 \mu \mathrm{L}$ of the polymer solution was mixed with $180 \mu \mathrm{L}$ of DTNB reagent solution in a microplate and after incubation of $15 \mathrm{~min}$ at RT the absorbance was measured at a wavelength of $405 \mathrm{~nm}$ (Spectra Max GeminiXS, Molecular Devices, CA, USA). Cysteine standard solutions were used to calculate the amount of free thiol moieties in the polymer.

\subsection{BDNF encoding plasmid DNA ( $p D N A$ ) production and purification}

The pDNA used encoded for the BDNF protein under the cytomegalovirus promoter (pCMVBDNF, 7.7 kb, a kind offer from Prof. Joost Verhaagen, Netherlands Institute for Neuroscience) was produced in DH5 $\alpha$ competent $E$. coli strain and purified using an EndoFree plasmid Giga kit (Qiagen, Germany) following the manufacturer's instructions. Plasmid concentration and purity were assessed by UV spectroscopy (Nanodrop ND-1000, Thermo Fisher Scientific Inc., MA, USA) and the absorbance ratio achieved $(260 \mathrm{~nm} / 280 \mathrm{~nm})$ was between 1.8 and 2.0.

\subsection{HC fragment production, purification and modification}

The plasmid encoding for HC fragment was a kind offer from Prof. Neil Fairweather (King's College, UK) and was produced and purified as previously described [31]. The purified fragment was modified with a bifunctional $5 \mathrm{kDa}$ poly (ethylene glycol) (PEG) spacer (Jenkem Technology, China) bearing an N-hydroxysuccinimide (NHS) and a maleimide (MAL) end group, at a 2.5 $\mathrm{PEG} / \mathrm{HC}$ protein molar ratio. The amount of reactive MAL groups in the HC fragment was determined using the Ellman's assay [30] and found to be $1.5 \mathrm{~mol}$ PEG/HC. 


\subsection{Preparation and characterization of TMC-based nanoparticles}

TMCSH-based nanoparticles were prepared using a molar ratio of quaternized amine groups $(\mathrm{N})$ to DNA phosphate groups $(\mathrm{P})(\mathrm{N} / \mathrm{P})$ of 2 . As illustrated in Scheme 1, the nanoparticle core was formed by mixing, while vortexing, equal volumes of pDNA solution with TMCSH solution in $20 \mathrm{mM}$ HEPES buffered saline solution containing $5 \%$ (w/v) glucose, pH 7.4. Complexes were let to stabilize for $15 \mathrm{~min}$ at RT and immediately frozen. After freeze-dried, nanoparticles were suspended in sterile distilled water (naked TMCSH nanoparticles) or in sterile distilled water containing the HC-PEG fragment in a w/w ratio of HC-PEG per pDNA of 4 (TMCSH-HC nanoparticles) and let to react for $24 \mathrm{~h}$ under agitation (RT). Both nanoparticles were characterized in terms of size, polydispersity index (Pdi) and zeta potential using a Zetasizer Nano Zs (Malvern, UK). The Smoluchowski model was applied for zeta potential determination and cumulant analysis was used for Z-average particle size determination. Ten $\mu \mathrm{g}$ of pDNA was used to prepare the tested formulations. All measurements were performed in triplicate at 25 $\stackrel{\circ}{ } \mathrm{C}$.

\subsection{In vitro transfection of dissociated DRG primary cultures}

All animal experiments described in this report were carried out with the permission of the local animal ethical committee in accordance with the EU Directive (2010/63/EU) and Portuguese law (DL 113/2013). The experimental protocol (reference 421/000/000/2013) was approved by the ethics committee of the Portuguese official authority on animal welfare and experimentation (Direção Geral de Alimentação e Veterinária).

Primary embryonic rat DRG neurons were isolated from Wistar embryo rats (E18) as described elsewhere [12]. From the final cell suspension, $5 \times 10^{4}$ cells $/ \mathrm{cm}^{2}$ of viable cells were seeded in a 24-well plate on glass coverslips (12 mm, MGF-Slides, Immuno-Cell, Belgium) pre-treated with $0.01 \mathrm{mg} / \mathrm{ml}$ of poly (D-lysine) (PDL, molecular weight 30-70 kDa, Sigma-Aldrich Co) and, subsequently, with $5 \mu \mathrm{g} / \mathrm{ml}$ laminin-1 isolated from mouse Engelbreth-Holm-Swarm sarcoma (Sigma-Aldrich Co), in DMEM/Ham's F12 with GlutaMAX ${ }^{\mathrm{TM}}, 50 \mu \mathrm{g} / \mathrm{ml}$ of penicillin and streptomycin, $25 \mu \mathrm{g} / \mathrm{ml}$ fungizone, $2 \%$ (v/v) B27 supplement (all from Gibco ${ }^{\circledR}$, Thermo Fisher Scientific Inc.) and $50 \mathrm{ng} / \mathrm{ml}$ of nerve growth factor (NGF) 7S (Calbiochem ${ }^{\circledR}$, Millipore, France). This protocol allowed a neuron purity $>70 \%$ at $48 \mathrm{~h}$ post-seeding [12]. At 3 days post-seeding dissociated DRG primary cultures were incubated for $6 \mathrm{~h}$ with naked BDNF encoding plasmid DNA (pBDNF), TMCSH/pBDNF or TMCSH-HC/pBDNF nanoparticles. Untreated cultured cells were used as control. Seventy-two hours after transfection the relative expression of BDNF mRNA in DRG dissociated primary cultures was determined by reverse transcription and quantitative real-time PCR (RT-qPCR) (see section 2.12 for experimental details).

\subsection{Nanoparticle administration and sciatic nerve crush injury}

A total of 48 young adult female BALB/c mice (10 weeks old) were used in this study. Animals were randomly divided into four groups as follows: (A) Vehicle group (received 20 mM HEPES buffer containing $5 \%(\mathrm{w} / \mathrm{v})$ glucose, $\mathrm{pH} 7.4, \mathrm{n}=15)$, (B) TMCSH group (received TMCSH nanoparticles in $20 \mathrm{mM}$ HEPES buffer containing 5\% (w/v) glucose, $\mathrm{pH} 7.4, \mathrm{n}=15$ ), (C) TMCSHHC group (received HC-functionalized TMCSH nanoparticles in 20 mM HEPES buffer containing $5 \%(\mathrm{w} / \mathrm{v})$ glucose, $\mathrm{pH} 7.4, \mathrm{n}=15)$ and (D) Wild-type group (uninjured animals used for mRNA quantification, $\mathrm{n}=3$ ).

Eight days before nerve crush, animals were injected with either $50 \mu \mathrm{L}$ of the vehicle solution or nanoparticle suspension (containing $50 \mu \mathrm{g}$ of BDNF pDNA) in the left medial gastrocnemius muscle under volatile anesthesia with isoflurane $(5 \%(\mathrm{v} / \mathrm{v})$ isoflurane $+0.8 \mathrm{l} / \mathrm{min}$ oxygen for 
induction; $2 \%-2.5 \%(\mathrm{v} / \mathrm{v})$ isoflurane $+0.2 \mathrm{l} / \mathrm{min}$ oxygen for maintenance). After injection, the needle was left in place for $1 \mathrm{~min}$ to avoid leakage.

Eight days later, animals were subjected to a unilateral crush injury in the left sciatic nerve, as we previously reported [32]. Animals were anesthetized with isoflurane, as described above, and shaved in the left hind limb. After skin incision, the left sciatic nerve was exposed and crushed twice within a $15 \mathrm{~s}$ period, using a Micro-Mosquito hemostat (Fine Science Tools GmbH, Germany). To standardize the procedure and yield reproducible sensorimotor deficits, the crush site was maintained constant for each animal. The skin incision was then closed with absorbable $6 / 0$ poly (glycolic acid) suture (Surgicryl PGA, SMI, Belgium). After surgery and twice a day (for $48 \mathrm{~h}$ ) animals received $0.05 \mathrm{mg} / \mathrm{kg}$ buprenorphine as analgesic. Animals were maintained in study during the following 21 days.

\subsection{Sensorimotor recovery analysis}

The sensorimotor recovery of animals from each treatment group was evaluated once a week throughout the study, namely before nanoparticle treatment (pre-treatment) and at 1, 7,14 and 21 days after sciatic nerve injury.

The locomotor function recovery was evaluated by the walking tract analysis. In brief, mice footprints were recorded by dipping the mice paws in ink and having the animals walking unassisted along a $60 \times 11 \times 6 \mathrm{~cm}$ corridor lined on the bottom with a sheet of white paper. The obtained tracts were then used to calculate the sciatic functional index (SFI) using the empirical equation adapted for mice by Inserra et al. [33]: SFI = 118.9 x ((ETS-CTS)/CTS) - $51.2 \times$ ((EPLCPL)/CPL) - 7.5, where ETS stands for operated experimental toe spread (distance between the first and fifth toes), CTS for control toe spread, EPL for operated experimental print length and CPL for control print length. The SFI scores vary among -100 (representing a non-functional nerve) and 0 (representing normal nerve function or completely nerve recovery). The pretreatment SFI values were used as control for comparison.

The sensory nociceptive function recovery of sciatic nerve was evaluated by the hot plate test through which the response to a noxious thermal stimulus is measured. This test is based on the measurement of the latency time before a withdrawal reflex when the mice feet are placed on a standard temperature-controlled plate. Mice were placed within a transparent Plexiglas box (12 x $22.5 \mathrm{~cm}$ ) on a standard temperature-controlled metal plate (hot plate analgesia meter, IITC Life Science, CA, USA) at $52{ }^{\circ} \mathrm{C}$, and a 30 s cut-off time was used for nonresponsive mice. The latency time to respond with a hind paw lick or shake/jump was measured and used as an index of nociception. To prevent sensitization, animals were subject to test only once per time point. The pre-treatment latency time was used as control for comparison.

The mechanical allodynia was measured using a series of calibrated Von Frey filaments (Ugo Basile, Italy), through the up-down method as described elsewhere [34] with minor modifications. Animals were placed in an opaque cage over a wire mesh floor and let to adapt to the testing environment for at least $20 \mathrm{~min}$. A set of ten calibrated von Frey filaments (ranging from 0.008 to $2 \mathrm{~g}$ of target force) were applied to the mid-plantar surface of the hind paw until they bent. This procedure was repeated 5 times with each filament and the threshold force (filament) that resulted in paw withdrawal was recorded. The contralateral paws were used to assess the uninjured paw withdrawal threshold.

\subsection{Motor nerve conduction velocity (MNCV)}

MNCV was determined at 21 days after sciatic nerve injury as previously described by Ferdinandusse et al. [35] with a Powerlab 4/25 (ADInstruments, UK) using the Chart5 software. 
The left sciatic nerve was stimulated by placing the stimulator electrode (monopolar needle, 29G, surgical steel) first at the sciatic notch (proximal stimulation) and then at the ankle (distal stimulation). The recording electrode was placed in the footpad. MNCV was calculated as the ratio of the distance between both sites of stimulation divided by the difference between proximal and distal latencies. The MNCV is expressed in meters per second (m/s). The MNCV of the contralateral uninjured sciatic nerves were used as control.

\subsection{Tissue processing and histological analysis}

Twenty nine days after nanoparticle administration, animals were anesthetized by intraperitoneal administration of sodium pentobarbital $(50 \mathrm{mg} / \mathrm{kg})$ and intracardially perfused with phosphate buffered saline (PBS; $1 \mathrm{mM} \mathrm{KH}_{2} \mathrm{PO}_{4} ; 5.6 \mathrm{mM} \mathrm{Na}_{2} \mathrm{HPO}_{4} ; 154 \mathrm{mM} \mathrm{NaCl}$ ) followed by freshly prepared $4 \%(\mathrm{w} / \mathrm{v}$ ) paraformaldehyde in $0.1 \mathrm{M}$ phosphate buffer, $\mathrm{pH}$ 7.4. The ipsiand contralateral sciatic nerves, lumbar DRG and the L3-L5 lumbar segments of spinal cord were collected and post-fixed in the same fixative overnight at $4{ }^{\circ} \mathrm{C}$. Gastrocnemius muscles were collected after PBS perfusion, weighted and fixed in $4 \%(\mathrm{w} / \mathrm{v})$ paraformaldehyde in $0.1 \mathrm{M}$ phosphate buffer, pH 7.4. Muscles used for muscle fiber morphological analysis were embedded in paraffin and the ones used for BDNF immunohistochemistry and neuromuscular junctions (NMJs) reinnervation analysis, as well as the remaining tissues, were cryopreserved in a $30 \%$ (w/v) sucrose solution and sectioned in a cryostat (HM550, Microm).

Immunofluorescence (IF) analysis. Frozen sections of gastrocnemius muscles (50 $\mu$ m-thick), sciatic nerves (10 $\mu \mathrm{m}$-thick), DRGs $(10 \mu \mathrm{m}$-thick) and spinal cord (16 $\mu \mathrm{m}$-thick) were immunostained for the detection of BDNF protein expression. Sciatic nerves were also immunostained for the detection of neurofilament-H (NF-H), growth-associated protein GAP43 (both neuronal cell markers) and S100 protein (Schwann cell marker) expression. Briefly, tissue sections were blocked and permeabilized for $1 \mathrm{~h}$ at RT with $5 \%(\mathrm{v} / \mathrm{v})$ normal goat serum in PBS containing $0.3 \%(\mathrm{v} / \mathrm{v})$ Triton X-100. Afterwards, tissue sections were incubated with primary antibody solution overnight at $4{ }^{\circ} \mathrm{C}$. After washing in PBS, tissue sections were incubated with fluorescently-labeled secondary antibody solution for $1 \mathrm{~h}$ at RT. Nuclei were stained with $0.1 \mathrm{mg} / \mathrm{ml} \mathrm{40,6-diamidino-2-phenylindole} \mathrm{(DAPI,} \mathrm{Sigma-Aldrich} \mathrm{Co).} \mathrm{The} \mathrm{primary}$ antibodies used were: polyclonal rabbit anti-BDNF antibody (Santa Cruz Biotechnology, CA, USA; 1:50), monoclonal mouse anti-NF-H antibody (Santa Cruz Biotechnology; 1:100), polyclonal rabbit anti-GAP-43 Alexa Fluor ${ }^{\circledR} 488$ conjugate (Novus Biologicals, Abingdon, UK; 1:100) and polyclonal rabbit anti-S100 antibody (Dako, Denmark; 1:200). As secondary antibodies were used a goat anti-mouse Alexa Fluor ${ }^{\circledR} 568$ conjugate and goat anti-rabbit Alexa Fluor ${ }^{\circledR} 488$ conjugate diluted in blocking buffer (all from Molecular Probes ${ }^{\circledR}$, Thermo Fisher Scientific Inc; 1:1000).

The semi-quantitative expression of BDNF, NF-H, GAP-43 and S100 protein in the sciatic nerves was assessed in the proximal, injury and distal segments of 3 sciatic nerve sections/animal, using the ImageJ 1.49t software (NIH, USA, http://imagej.nih.gov/ij/). The contralateral sciatic nerves were used as uninjured control. Results shown represent the area occupied by pixels corresponding to the fluorescent signal, normalized in relation to the total area.

The semi-quantitative expression of BDNF in lumbar DRGs (L4 and L5) and in the L3-L5 lumbar segments of spinal cord was assessed in 3 tissue sections/animal using a custom made program written in MATLAB (The MathWorks, version 2015a, Natick MA, USA). The contralateral L4 and L5 DRGs and the contralateral (right) spinal cord hemisection were used as uninjured controls, respectively. Results were expressed as mean pixel intensity levels (fluorescence). 
The analysis of NMJ reinnervation was performed in 50 mmthick frozen sections of gastrocnemius muscle through a double staining for the postsynaptic marker $\alpha$-bungarotoxin and the neurofilament 200 (NF200) marker. In brief, tissue sections were blocked and permeabilized for $1 \mathrm{~h}$ at RT with $5 \%(\mathrm{v} / \mathrm{v})$ normal goat serum in PBS containing $0.3 \%(\mathrm{v} / \mathrm{v})$ Triton X-100 and incubated with the primary polyclonal rabbit anti-NF200 antibody (Abcam, Cambridge, UK; $1: 500$ ) overnight at $4 \stackrel{\circ}{\circ}$. After washing in PBS, tissue sections were incubated with a secondary antibody goat anti-rabbit Alexa Fluor ${ }^{\circledR} 488$ conjugate and $\alpha$-bungarotoxin e tetramethylrhodamine conjugate diluted in blocking buffer for $90 \mathrm{~min}$ at RT (both from Molecular Probes ${ }^{\circledR}$, Thermo Fisher Scientific Inc.; 1:1000 and 1:200, respectively). The fraction of denervated, partial and complete reinnervated NMJ was counted in Z-stack confocal microscopy images using the maximum intensity projection. The innervation status of individual NMJ was classified according to the extent to which the neurofilament labeling overlaid with $\alpha$-bungarotoxin staining and was presented as relative to $100 \%$ (referent to a tissue where all NMJ are completely innervated (uninjured)). Denervated NMJs were devoid of any NF200 labeling. Partial innervated NMJs were identified as only part of the $\alpha$-bungarotoxin positive endplates being covered by NF200 marker labeling. Completely innervated NMJs were defined by the complete overlap of NF200 and $\alpha$-bungarotoxin labeling. For each muscle sample at least $50 \mathrm{NMJ}$ were evaluated from randomly selected visual fields.

For morphological analysis and quantification of cross sectional muscle fibers area, transversal paraffin sections of ipsi- and contralateral gastrocnemius muscles ( $3 \mu \mathrm{m}$-thick) were stained with hematoxylin and eosin (H\&E). A total of 40 muscle fibers per animal was randomly selected and used to quantify the cross sectional area (20 fibers from lateral and 20 fibers from medial gastrocnemius muscle). The quantification was performed using the Image $1.49 \mathrm{t}$ software (NIH, USA, http://imagej.nih.gov/ij/). The contralateral gastrocnemius muscles were used as uninjured control.

\subsection{Gastrocnemius muscle wet weight}

The ratio between the wet weight of the gastrocnemius muscle from the experimental side and the contralateral side (uninjured) was used to indirectly assess the target muscle innervation recovery. Twenty-one days post-injury, after PBS perfusion, the ipsi- and contralateral gastrocnemius muscles were dissected and weighed.

\subsection{Myelinated vs. unmyelinated axon quantification by transmission electron microscopy (TEM)}

After perfusion, distal sciatic nerve segments of 5 animals/group were harvested and post-fixed in $2.5 \%(\mathrm{w} / \mathrm{v})$ glutaraldehyde and $2 \%(\mathrm{w} / \mathrm{v})$ paraformaldehyde diluted in $0.1 \mathrm{M}$ sodium cacodylate for $4 \mathrm{~h}$. The nerve segments were subsequently post-fixed in $1 \%(\mathrm{v} / \mathrm{v})$ osmium tetroxide for $2 \mathrm{~h}$ and dehydrated in series of graded ethanol. Tissues were embedded in Spurr resin (EMS- Electron Microscopy Sciences, Hatfield, PA, USA) in a $60{ }^{\circ} \mathrm{C}$ oven for $48 \mathrm{~h}$. Semi-thin sections $(1-2 \mu \mathrm{m})$ were stained with $0.01 \%(\mathrm{w} / \mathrm{v})$ toluidine blue, $0.05 \%(\mathrm{w} / \mathrm{v})$ methylene blue and $0.05 \%(\mathrm{w} / \mathrm{v})$ borax in aqueous solution. Afterwards, the density of myelinated axons was counted in the whole nerve area. Regarding unmyelinated axons, ultra-thin transverse sciatic nerve sections $(60-90 \mathrm{~nm})$ were cut and stained with $2 \%(\mathrm{w} / \mathrm{v})$ uranyl acetate in aqueous solution. The stained samples were mounted on mesh grids and observed under a transmission electron microscope (JEM-1400EX; JEOL Ltd., Tokyo, Japan). For each animal, the density of unmyelinated axons was counted in 50 random and non-overlapping photographs (magnification 12000x). 


\subsection{One-step RT-qPCR}

At $72 \mathrm{~h}$ after transfection, the dissociated DRG primary cultures were gently trypsinized, collected, washed with cold PBS and placed on ice to be freshly processed (not frozen). Total RNA was extracted and purified from cultured cells using the mirVana ${ }^{\mathrm{TM}}$ miRNA Isolation Kit (LifeTechnologies, CA, USA) with DNase digestion.

For the in vivo studies, 3 animals/group were sacrificed by a lethal intraperitoneal injection of $20 \%$ (v/v) sodium pentobarbital and tissues were collected and snap frozen. Total RNA was extracted and purified from frozen tissues using the Quick-RNA ${ }^{\mathrm{TM}}$ Miniprep kit (Zymo Research Corp., CA, USA) with DNase I treatment.

For detection of BDNF mRNA levels, $10 \mathrm{ng}$ of purified RNA was used per sample and amplified in duplicate in an iCycler iQ5 (BioRad, CA, USA) using the SYBR Green One-step qPCR Kit (Biotool. com, Germany), according to the manufacturer's instructions. The glyceraldehyde-30 phosphate dehydrogenase (GAPDH) mRNA levels were used as an internal control to normalize the variability in expression levels. The sequences of the primers used for BDNF gene were $5^{\prime}$ TGGCTGACACTTTTGAGCAC-3' and 5'-GTTTGCGGCATCCAGGTAAT-3' and for GAPDH gene were 5'-GCCTTCCGTGTTCCTACC-3' and 5'-AGAGTGGGAGTTGCTGTTG-3'. At the end of the PCR cycling, correspondent melting curves were performed in order to ascertain the amplification of a single product and the absence of primer dimmer formation. The normalized BDNF expression levels in treatment groups were calculated relative to untreated cultured cells (in vitro transfection studies) or to the uninjured wild-type group (in vivo studies) using the 2- $\Delta \Delta \mathrm{CT}$ relative quantification method described by Livak and Schmittgen [36].

\subsection{Statistical analysis}

Statistical analysis was performed using GraphPad Prism version 6.0 for Windows (San Diego California, USA). Results are presented as mean \pm standard deviation (SD). Significant differences between treatment groups were determined by using the ordinary one-way ANOVA test followed by the Newman-Keuls post-test for multiple comparisons. The one sample $t$-test was used to compare the mean BDNF mRNA expression levels (RT-qPCR analysis) between treatments groups and wild-type animals. Results with $\mathrm{p}<0.05$ were considered statistically significant.

\section{Results}

\subsection{Characterization of TMCSH-based nanoparticles}

The prepared TMCSH-based nanoparticles were characterized in terms of size, Pdi and zeta potential in $20 \mathrm{mM}$ HEPES buffer containing $5 \%(\mathrm{w} / \mathrm{v})$ glucose, $\mathrm{pH} 7.4$ As shown in Table 1, the mean size diameter and Pdi values obtained for TMCSH-HC nanoparticles were not significantly different from the TMCSH ones, showing that HC-functionalization does not influence the average size and polydispersity of the nanoparticles. Although significantly lower for the TMCSH-HC, zeta potential values obtained for both nanoparticles were positive, indicating a cationic surface charge of both nanoparticles with a good colloidal stability.

\subsection{Sensorimotor functional recovery}

All animals under study were frequently checked for weight variation and autotomy. After sciatic nerve injury, rodents frequently scratch and bite theirs anesthetized foot which ultimately can result in autotomy of one or more toes, usually observed in the beginning of the 
third post-injury week [37]. No weight variation and autotomy were observed in all groups in study, but 3 out of 15 total animals of vehicle group showed nibbling of one or two toenails. Additionally, 2 and 1 out of 15 total animals of TMCSH and TMCSH-HC treatment groups, respectively, showed some swelling of one or two toes, with no apparent lesion. Even so, these animals did not reveal a painful behavior and their locomotor activity was not altered.

The locomotor functional recovery of animals in each treatment group was monitored once a week by determining the sciatic functional index (SFI). Based on SFI values, one can infer crucial aspects of locomotor activity involving the recovery of hind limb sensory and motor function after an injury. Fig. 1A displays the changes in the SFI values as a function of time for the three treatment groups. The preoperative SFI scores are indicative of normal nerve function. Postoperative SFI scores sharply decreased, oscillating between -90 and -80 during the first week, due to injury-induced loss of function, and start to gradually increase during the following weeks, reflecting a functional recovery in all groups under investigation. At the end of the study all groups showed a marked recovery, reaching SFI values around -20. No significant differences were observed between groups.

The thermal nociceptive sensory function in response to a noxious thermal stimulus was measured by the hot plate assay. Seven days after nerve injury, all treatment groups showed a delay in response to the thermal stimulus that was progressively recovered in the subsequent time points of the study (Fig. 1B). At 21 days after crush injury, the latency time to respond to the thermal stimulus was significantly lower in the TMCSH and TMCSH-HC groups when compared to the control vehicle group, reaching similar values to the pre-treatment time-point. In contrast, the latency time response of animals in the control vehicle was significantly different between 21 days after injury and the pre-treatment time-point. Moreover, animals belonging to the TMCSH-HC group showed a significantly quicker response than the ones in the TMCSH group.

The mechanical nociceptive sensory function was assessed with the Von Frey filaments stimulation (Fig. 1C). Mechanical allodynia was observed in the period span of the study in all treatment groups, starting as soon as 7 days after nerve injury for the TMCSH treated animals. At this time point, animals from the vehicle and TMCSH-HC treatment groups showed no indication of mechanical allodynia. In the TMCSH-HC group, mechanical threshold values were unchanged in comparison to the contralateral values while in the vehicle group an increase in the mechanical threshold values were observed although not significantly different from the contralateral values.

The electrophysiological assessments of the motor nerve conduction velocity (MNCV) performed at 21 days after nerve injury provided further evidence for the functional recovery of the crushed sciatic nerves. Although the MNCV values in each treatment group were significantly lower than those recorded in the contralateral uninjured nerves (Fig. 1D), animals treated with TMCSH-HC nanoparticles showed a significant elevation in their MNCV values when compared to the control vehicle group.

\subsection{BDNF biodistribution}

At the end of the study, the expression levels of BDNF mRNA were determined in the left gastrocnemius muscle, injured sciatic nerve, ipsilateral lumbar DRGs and lumbar spinal cord and expressed as relative to levels in the respective tissue in wild-type animals (uninjured and untreated) (Fig. 2). In the gastrocnemius muscle, one observed a significant decrease of BDNF mRNA levels in the vehicle group as compared to wild-type animals while in the TMCSH group those were maintained unchanged (Fig. 2A). Additionally, the TMCSH-HC group showed a significantly higher expression of BDNF mRNA than those presented in vehicle and TMCSH 
treatment groups, although not significantly different from the one of wild-type animals (Fig. 2A). In the ipsilateral lumbar DRGs, the BDNF mRNA expression levels were similar between the TMCSH group and wild-type animals (Fig. 2B). In contrast, the BDNF mRNA expression was significantly decreased in the vehicle group and increased in the TMCSH-HC treatment group as compared to wild-type animals (Fig. 2B). Moreover, the mRNA expression levels in the TMCSHHC group were significantly higher than the BDNF mRNA levels expressed in the animals treated with the vehicle solution (Fig. 2B). These results are in line with our in vitro dissociated DRG primary culture transfection data that show a significant increase in the BDNF mRNA expression levels in DRG cultures treated with the TMCSH-HC nanoparticles as compared to the TMCSH nanoparticles or naked plasmid (see Fig. S3 in Supplementary Information). In the ipsilateral sciatic nerves and lumbar spinal cords the BDNF mRNA levels were similar between all treatment groups and wild-type animals, with no significant differences being observed between treatment groups (Fig. 2C and D, respectively).

The BDNF protein expression levels were also evaluated in the same tissues at 21 days after injury (Fig. 3). In the gastrocnemius muscle, the BDNF protein was not immuno detected in any of the treatment groups (data not shown). Contrarily, BDNF was detected in sciatic nerves of animals from all treatment groups (Fig. 3A-D). However, solely animals treated with TMCSH-HC nanoparticles revealed a significant increase in the BDNF protein expression when compared to the basal contralateral (uninjured nerve) BDNF expression levels (Fig. 3E). Similarly, animals treated with TMCSHHC were the ones displaying higher levels of BDNF protein in the lumbar DRGs and spinal cord (Fig. 3F-J and K-N, respectively), being these significantly different from the basal contralateral (uninjured) BDNF expression levels in the case of the DRGs.

\subsection{Histological analysis of crushed sciatic nerves}

At 21 days after sciatic nerve crush injury, nerves from the different treatment groups were immunostained for the detection of NF-H, GAP-43 protein and the S100 Schwann cell marker.

Axonal protection and regeneration was investigated through the staining of neurofilament NF$\mathrm{H}$ and the growth-associated protein GAP-43 positive axons. Results showed that the amount of NF-H positive nerve axons were progressively lower along the crushed sciatic nerves - from the proximal to the distal stump - in the vehicle and TMCSH treated groups (Fig. 4A-D and M).

Contrarily, 21 days after injury the levels of NF-H positive axons in the TMCSH-HC group were similar in all analyzed nerve segments, being these significantly higher than those expressed in both the vehicle and TMCSH groups at the distal nerve segment (Fig. 4A-D and M). The levels of GAP-43 protein were generally increased in all nerve segments of all treatment groups as compared to the GAP-43 levels of the contralateral uncrushed nerves. In the control vehicle group, the expression of GAP-43 protein was progressively reduced in nerve segments reaching similar levels to the contralateral nerves in the distal nerve stump (Fig. 4E-H and N). This expression levels were significantly lower than those present in the distal nerve segments of animals treated with TMCSH and TMCSH-HC nanoparticles.

Schwann cells response to injury was evaluated by the immunostaining of sciatic nerves with the S100 protein marker. Fig. 40 points to a significant increase in the S100 protein expression levels, as compared to those in the contralateral uninjured tissue, in the nerve proximal stump for all treatment groups and in the nerve injury region for the TMCSH-HC treatment. Although not statistically significant, the levels of S100 protein expression in the distal portion of sciatic nerve were higher for animals treated with both nanoparticle formulations (Fig. 4I-L and O), evidencing a slight improvement in Schwann cell proliferation. 
Following a nerve crush injury, axonal damage, demyelination and subsequent re-myelination of the regenerating axons occur sequentially in time. Consequently, the degree of axonal remyelination observed after an injury can be used as an indicator of nerve recovery. To assess the re-myelination extent after injury, transverse semi-thin sections of the distal nerve segment were analyzed by toluidine blue staining (Fig. 5A-D). Results showed a large number of well myelinated axons, with thick myelin sheaths, in the TMCSH and TMCSH-HC groups (Fig. 5C-D). In contrast, sciatic nerve axons treated with vehicle solution exhibited thinner myelin sheaths and several degenerating axons (Fig. 5B). The quantification of myelinated axons per surface area showed a significantly higher density of myelinated axons in animals treated with TMCSHHC nanoparticles, being these values similar to the ones found for the uninjured contralateral nerves (Fig. 5I). We also evaluated the extent of the regeneration of unmyelinated axons in transverse ultra-thin sections of the distal nerve segment by TEM (Fig. 5E-H). Sciatic nerves from vehicle group showed many Remak bundles (group of unmyelinated axons) containing a number of very tightly grouped unmyelinated axons of different sizes surrounded by a single Schwann cell. In contrast, sciatic nerves from TMCSH and TMCSH-HC groups presented less compact Remak bundles being the unmyelinated axons relatively homogenous in size, similar to the contralateral uninjured nerves (Fig. 5E). The density of unmyelinated axons in the nanoparticle treated animals was found to be similar to the one observed in uninjured animals, independently of the formulation (targeted vs. non-targeted) (Fig. 5J). In opposition, animals in the vehicle treatment group showed a significant increase, of approximately 59\%, in the density of unmyelinated axons (Fig. 5J).

\subsection{Reinnervation of target gastrocnemius muscle}

Hematoxylin and eosin stained sections of gastrocnemius muscles after 21 days post-injury were used to assess the reinnervation of the denervated gastrocnemius muscle on the injured side (Fig. 6A-D). The gastrocnemius muscle in all treatment groups displayed signs of muscle denervation, with a maximal muscle mass loss of $35 \%$ being observed in the vehicle group, as compared to the contralateral normal muscle (Fig. 6E). In the vehicle group muscle fibers revealed a considerable atrophy as compared to that in the other treatment groups. The myofibers lost their scattered distribution and were rearranged in groups of small rounded fibers separated from each other by a number of adipocytes (Fig. 6B). In both nanoparticle treatment groups, muscle fibers also presented a round-shape but were less shrunk than the ones observed in the vehicle treatment group. Few or none adipocytes were seen between myofibers. The morphometric analysis further indicated that the cross sectional area of muscle fibers in the three treatment groups showed significant differences compared to the contralateral normal gastrocnemius muscle. As shown in Fig. 6F, animals treated with nanoparticles showed a significant higher muscle fiber cross sectional area than the ones in vehicle group. The greater effect on the recovery of gastrocnemius muscle cross sectional area was observed in the TMCSH nanoparticle treatment group. These findings were further corroborated by the analysis of the degree of reinnervation of the denervated gastrocnemius muscle. At 21 days after sciatic nerve crush, double staining for the postsynaptic marker $\alpha$ bungarotoxin and the NF200 marker revealed a difference in the fraction of denervated and reinnervated NMJ between treatment groups. As shown in Fig. 6G, animals from the vehicle group showed a significantly higher percentage of denervated NMJ $(40.2 \pm 4.4 \%)$ as compared to the TMCSH and TMCSH-HC treated animals $(13.2 \pm 12.0 \%$ and $10.0 \pm 6.1 \%$, respectively). On the other hand, although not statistically significant, the percentage of completely innervated NMJ was higher in the TMCSH-HC group when compared to the TMCSH and vehicle groups. 


\section{Discussion}

The management of nerve lesions resulting from peripheral neuropathies remains a major clinical challenge. The pathological features of axonal degeneration occurring in the majority of human peripheral neuropathies are quite similar to Wallerian degeneration, being neuropathies commonly described as "Wallerian-like" degenerative conditions [38,39]. This similarity makes the experimental Wallerian degeneration models useful tools for studying the pathogenesis and the efficacy of potential treatments for peripheral neuropathies. In a scenario of Wallerian degeneration, Schwann cells, together with macrophages, respond by clearing the degenerated axons and myelin debris. Further, Schwann cells enter in a dedifferentiation process, starting to proliferate to form bands of Büngner, important for guiding the regrowing axons. Schwann cells also secrete neurotrophic factors and proteins of the extracellular matrix that promote the regeneration of the injured nerve. However, in severe and/or prolonged denervation conditions, Schwann cells are not capable of sustaining this process in time, limiting the ability of peripheral nerves to fully regenerate $[40,41]$.

Several studies have demonstrated that the administration of neurotrophic factors could aid preventing nerve degeneration and denervation of target tissues in the context of neuropathy, as well as promote nerve regeneration and consequent functional recovery of regenerating nerves [42]. But neurotrophic factors have a very short half-life being quickly cleared in vivo and, due to their pleiotropic actions, they can cause negative off-target effects. Motivated to contribute to the development of safe and targeted therapeutic strategies to promote peripheral nerve repair here we describe a non-viral, non-toxic and biodegradable nanocarrier based on TMC [28], designed to allow the targeted and efficient delivery and expression of a BDNF gene in peripheral neurons. The aim of the current study was to evaluate if the intramuscular administration of the developed neuron-targeted TMCSH-HC/BDNF nanoparticles in a nerve injury scenario would protect peripheral neurons from prolonged degeneration and/or enhance their regeneration ability.

Nanoparticles were intramuscularly administrated in the gastrocnemius muscle, a clinically relevant route of administration, 8 days before sciatic nerve crush injury. This nerve injury model is particularly helpful to study the effect of therapeutic agents for neuroprotection and regeneration, for the reason that it causes the rupture of nerve axons without destroying most of the nerve's supporting structures, i.e. endoneurial tubes, thereby providing a guide for axons to regenerate spontaneously and even partially reinnervate their targets, mimicking the observed series of events that can occur in an axonal neuropathy. Therefore, using this injury model, the success of a particular therapeutic agent is directly correlated with the evidence of axonal degeneration prevention and/or acceleration of nerve regeneration. The pre-injury administration would allow the nanoparticles to reach the neuron cell bodies before the injury [13], and in this way one could evaluate their potential to create a cellular milieu that would protect neurons from prolonged degeneration and/or enhance the regeneration process.

The treatment with TMCSH-HC nanoparticles significantly elevated the BDNF expression in neural tissues, as observed 21 days after injury. The BDNF mRNA levels were significantly increased in the lumbar DRGs, as compared to wild-type animals (uninjured) and vehicle group, being the respective protein expression levels also augmented in the lumbar DRGs, lumbar spinal cord segments and sciatic nerves. Taking into account these observations, we consider that the BDNF levels elevation observed in TMCSH-HC treated animals is a result of nanoparticle-mediated BDNF gene delivery in these tissues and not a response to the nerve injury itself.

Further we confirmed that BDNF delivery and expression within the neural tissues promoted a general recovery of injured peripheral nerves. Vehicle treated animals showed a progressive 
recovery of their locomotor activity, significant alterations in their thermal response latency throughout the study (thermal hypoalgesia), mechanical hypoalgesia in the first week postinjury and mechanical allodynia, which was evident within 2 weeks after nerve injury. In addition, vehicle treated animals showed a marked reduction of their motor nerve conduction velocities (MNCVs), which was correlated to the lower density of myelinated axons evidenced in this treatment group. In contrast, animals treated with targeted and non-targeted nanoparticles demonstrated a gradual recovery of their thermal nociceptive function, more evident for the TMCSH-HC group, reaching the preoperative response latency values at the end of the study. In the timeframe of the study, nanoparticle treatment did not prevented mechanical allodynia, but animals from the TMCSH-HC group showed a delay in the allodynia onset, with treated animals presenting similar paw withdrawal thresholds to the contralateral side at 7 days postinjury. No significant differences were observed in the locomotion activity recovery between all treatment groups. However, treatment with TMCSH-HC nanoparticles resulted in a significant increase in the MNCV in comparison with the vehicle group animals, although these values were still reduced as compared to the contralateral normal MNCV values. It is considered that noxious heat nociception is predominantly mediated by unmyelinated axons (C-fibers), organized as Remak bundles, whereas mechanical nociception and MNCV are mainly transmitted through the myelinated axons (A-fibers) [43]. Therefore, these sensorimotor functional results demonstrate that the BDNF delivery mediated by the targeted TMCSH-HC nanoparticles might have an effect in both myelinated and unmyelinated axons.

To determine the structural changes in both myelinated and unmyelinated axons of the sciatic nerve that may underlie the functional differences observed between treatment groups, a detailed morphometric study of the injured nerves was conducted 21 days post-injury (29 days post-nanoparticle treatment). A significant decrease in the myelinated axon density in the vehicle and TMCSH groups was observed, as compared to the contralateral uninjured nerves. In contrast, animals treated with TMCSH-HC nanoparticles presented similar density of myelinated axons to the contralateral uninjured nerves, being these values significantly higher than the ones presented by vehicle and TMCSH treated animals. Despite that the density of myelinated axons was close to the normal levels, TMCSH-HC treated nerves were not yet fully functional suggesting that the remyelination process was not completed at this time point of analysis. On the other hand, the density of unmyelinated axons appeared to be significantly unaffected in the animals treated with both nanoparticle formulations (targeted and nontargeted), justifying the great thermal nociceptive recovery observed in the nanoparticletreated animals. In opposition, animals in the vehicle treatment group showed a significant increase, of approximately 59\%, in the density of unmyelinated axons. These results are in line with previous reports showing that following a nerve crush injury unmyelinated axons branch in a tentative to search for a target [44]. Without a therapeutic intervention this branching can persist for a prolonged period of time until the axons reach the target tissues, an event that stops additional branching and triggers pruning to eliminate redundant branches. In accordance, we consider that, at the time point of analysis, unmyelinated axons of the vehicle treated animals might have not yet fully reached the target tissues and are still responding to the injury. In these conditions, unmyelinated axons are not totally functional, which is supported by the thermal hypoalgesia observed. These findings are corroborated by the analysis of the expression of the NF-H and GAP-43 markers. In contrast with animals from vehicle group, at this time point, TMCSH-HC treated animals exhibited similar levels of NF-H expression to the contralateral uninjured nerves and significantly augmented levels of GAP-43 protein, indicating that the original nerve structure has already recovered and regenerating axons were actively reinnervating their targets. 
Upon a sciatic nerve crush injury the target limb muscles become denervated. Previous studies have shown that, after denervation, muscle wet weight was found to dramatically decrease in the first days after injury, reaching a minimum level at about day 14, and then progressively recovering its mass to approximately $80 \%$ the weight of normal contralateral muscle by 28 days after nerve injury $[45,46]$. Our results are in concordance with the ones previously reported. Additionally, our data shows that animals treated with TMCSH and TMCSH-HC nanoparticles displayed a decrease in the muscle fiber atrophy, as well as a higher and earlier reinnervation of the NMJ in the gastrocnemius muscles, which seems to correlate with the nerve protection observed in these groups. This can also explain the normal levels of BDNF mRNA expression observed in both treatment groups 21 days after injury, which contrast with the reduced levels observed in the vehicle group.

Similarly to targeted nanoparticles, our study showed that non-targeted nanoparticles were also able to exert a beneficial effect in the injury recovery, mainly in what regards to target muscle recovery. We believe these nanoparticles, without targeting moieties, were preferentially retained at the injection site where they probably lead to the transient muscle cell transfection. Previous studies have shown that BDNF can potentiate in vivo muscle regeneration, through its stimulatory effect on muscle stem cells function [47], as well as nerve regeneration, acting as a retrograde signal for survival of innervating neurons via the TrkB receptor $[48,49]$. Thus, the local expression of BDNF may then have exerted its action locally, by autocrine signaling, or remotely, via paracrine signaling, in Schwann cells and/or in the distal portion of injured sciatic nerves, which resulted in the reversion of the target muscle amyotrophic status and in the improvement of sciatic nerve recovery as well. Despite that, the HC-functionalization of the nanoparticles increases the chance of nanoparticle binding and internalization in neural populations, leading to an improvement in the BDNF expression in neural tissues and consequent better sciatic nerve recovery. This highlights the superior efficacy of the targeted nanoparticles to be applied in the context of peripheral nerve injury.

Altogether, our findings indicate that the developed neuron-targeted TMCSH-HC nanoparticles are capable of mediating the delivery and subsequent expression of BDNF transgene in peripheral neurons after a peripheral intramuscular administration, providing the trophic support needed to allow injured neurons to more quickly respond to the injury and accelerate their regeneration and target muscle reinnervation. This neuroprotection and enhanced regeneration resulted in satisfactory sensorimotor functional recovery.

To the best of our knowledge this is the first study that reports the improvement of nerve regeneration as a result of a gene therapy treatment mediated by a non-viral vector. Based on our results, we believe that the intramuscular injection of the non-viral TMCSH-HC nanoparticles carrying therapeutic transgenes that encode for neurotrophic factors is an efficient and safe way of preventing prolonged nerve degeneration and, consequently, accelerate nerve regeneration. It should be highlighted that due to its safety profile these nanoparticles can also be foreseen for multiple administrations, to extend the envisaged therapeutic effect.

\section{Conclusions}

Aiming at developing alternative therapeutic strategies to treat peripheral neuropathies, in this study we introduced a versatile, safe and efficient neuron-targeted non-viral vector based on thiolated TMC to be used as gene delivery carrier. The ability of these nanoparticles to specifically mediate a therapeutic transgene delivery to peripheral neurons after a minimally 
invasive clinically relevant peripheral administration, and promote the recovery of a crushed nerve by preventing nerve degeneration and enhancing nerve regeneration was demonstrated.

This opens new avenues for the treatment of nerve injury deriving from peripheral neuropathies based on safe nanoparticle-mediated gene delivery strategies.

\section{Acknowledgments}

The work was financed by Portuguese funds through FCT - Fundação para a Ciência e a Tecnologia in the framework of the projects PTDC/CTM-NAN/115124/2009 and PTDC/CTMNAN/3547/2014. Cátia D. F. Lopes, Nádia P. Gonçalves and Carla P. Gomes acknowledge FCT for their Ph.D. scholarships (SFRH/BD/77933/2011, SFRH/BD/74304/2010 and SFRH/BD/77930/2011, respectively). Authors further acknowledge the Biointerfaces and Nanotechnology Service of i3S for the nanoparticle characterization studies, Centro de Materiais da Universidade do Porto (CEMUP) for NMR analysis and Bioimaging Center for Biomaterials and Regenerative Therapies (b.IMAGE) for the confocal microscopy. The contribution of Dr. Paulo Aguiar (INEB|i3S) to MATLAB programing is also acknowledged.

\section{References}

[1] J.C. Watson, P.J. Dyck, Peripheral neuropathy: a practical approach to diagnosis and symptom management, Mayo Clin. Proc. 90 (7) (2015) 940e951.

[2] J.R. Goss, The therapeutic potential of gene transfer for the treatment of peripheral neuropathies, Expert Rev. Mol. Med. 9 (8) (2007) $1 \mathrm{e} 20$.

[3] S.C. Apfel, S. Schwartz, B.T. Adornato, R. Freeman, V. Biton, M. Rendell, A. Vinik, M. Giuliani, J.C. Stevens, R. Barbano, P.J. Dyck, Efficacy and safety of recombinant human nerve growth factor in patients with diabetic polyneuropathy: a randomized controlled trial. rhNGF Clinical Investigator Group, JAMA 284 (17) (2000) 2215e2221.

[4] A. Wellmer, V.P. Misra, M.K. Sharief, P.G. Kopelman, P. Anand, A double-blind placebo-controlled clinical trial of recombinant human brain-derived neurotrophic factor (rhBDNF) in diabetic polyneuropathy, J. Peripher Nerv. Syst. 6 (4) (2001) 204e210.

[5] J.F. Poduslo, G.L. Curran, Permeability at the blood-brain and blood-nerve barriers of the neurotrophic factors: NGF, CNTF, NT-3, BDNF, Brain Res. Mol. Brain Res. 36 (2) (1996) 280e286.

[6] J.F. Poduslo, G.L. Curran, J.S. Gill, Putrescine-modified nerve growth factor: bioactivity, plasma pharmacokinetics, blood-brain/nerve barrier permeability, and nervous system biodistribution, J. Neurochem. 71 (4) (1998) 1651e1660.

[7] H.U. Saragovi, K. Gehring, Development of pharmacological agents for targeting neurotrophins and their receptors, Trends Pharmacol. Sci. 21 (3) (2000) 93e98.

[8] J.C. Glorioso, M. Mata, D.J. Fink, Therapeutic gene transfer to the nervous system using viral vectors, J. Neurovirol. 9 (2) (2003) 165e172.

[9] A.P. Pêgo, H. Oliveira, P. Moreno, Biomaterial-based vectors for targeted delivery of nucleic acids to the nervous system, in: J. Coelho (Ed.), Drug Delivery Systems: Advanced Technologies Potentially Applicable in Personalised Treatment, Springer Netherlands, 2013, pp. 185e224.

[10] C.P. Gomes, C.D.F. Lopes, P.M.D. Moreno, A. Varela-Moreira, M.J. Alonso, A.P. Pêgo, Translating chitosan to clinical delivery of nucleic acid-based drugs, MRS Bull. 39 (01) (2014) 60e70.

[11] M. Thanou, B.I. Florea, M. Geldof, H.E. Junginger, G. Borchard, Quaternized chitosan oligomers as novel gene delivery vectors in epithelial cell lines, Biomaterials 23 (1) (2002) 153e159.

[12] C.D. Lopes, C.P. Gomes, E. Neto, P. Sampaio, P. Aguiar, A.P. Pêgo, Microfluidic-based platform to mimic the in vivo peripheral administration of neurotropic nanoparticles, Nanomedicine (Lond.) 11 (24) (2016) 3205e3221.

[13] C.D. Lopes, H. Oliveira, I. Estevao, L.R. Pires, A.P. Pêgo, In vivo targeted gene delivery to peripheral neurons mediated by neurotropic poly(ethylene imine)-based nanoparticles, Int. J. Nanomedicine 11 (2016) 2675e2683. 
[14] J.Y. Zhang, X.G. Luo, C.J. Xian, Z.H. Liu, X.F. Zhou, Endogenous BDNF is required for myelination and regeneration of injured sciatic nerve in rodents, Eur. J. Neurosci. 12 (12) (2000) 4171e4180.

[15] T. Fukuoka, E. Kondo, Y. Dai, N. Hashimoto, K. Noguchi, Brain-derived neurotrophic factor increases in the uninjured dorsal root ganglion neurons in selective spinal nerve ligation model, J. Neurosci. 21 (13) (2001) 4891e4900.

[16] H. Kobayashi, M. Yokoyama, Y. Matsuoka, M. Omori, Y. Itano, R. Kaku, K. Morita, H. Ichikawa, Expression changes of multiple brain-derived neurotrophic factor transcripts in selective spinal nerve ligation model and complete Freund's adjuvant model, Brain Res. 1206 (2008) 13e19.

[17] G. Miletic, V. Miletic, Increases in the concentration of brain derived neurotrophic factor in the lumbar spinal dorsal horn are associated with pain behavior following chronic constriction injury in rats, Neurosci. Lett. 319 (3) (2002) 137e140.

[18] S.J. Geng, F.F. Liao, W.H. Dang, X. Ding, X.D. Liu, J. Cai, J.S. Han, Y. Wan, G.G. Xing, Contribution of the spinal cord BDNF to the development of neuropathic pain by activation of the NR2Bcontaining NMDA receptors in rats with spinal nerve ligation, Exp. Neurol. 222 (2) (2010) 256e266. [19] E. Foster, B. Robertson, K. Fried, trkB-like immunoreactivity in rat dorsal root ganglia following sciatic nerve injury, Brain Res. 659 (1e2) (1994) $267 \mathrm{e} 271$.

[20] H. Hammarberg, F. Piehl, M. Risling, S. Cullheim, Differential regulation of trophic factor receptor mRNAs in spinal motoneurons after sciatic nerve transection and ventral root avulsion in the rat, J. Comp. Neurol. 426 (4) (2000) 587e601.

[21] R. Gauthier, S. Joly, V. Pernet, P. Lachapelle, A. Di Polo, Brain-derived neurotrophic factor gene delivery to muller glia preserves structure and function of light-damaged photoreceptors, Investig.Investig. Ophthalmol. Vis. Sci. 46 (9) (2005) 3383e3392.

[22] R. Ren, Y. Li, Z. Liu, K. Liu, S. He, Long-term rescue of rat retinal ganglion cells and visual function by AAV-mediated BDNF expression after acute elevation of intraocular pressure, Investig.Investig. Ophthalmol. Vis. Sci. 53 (2) (2012) 1003e1011.

[23] C. Wan, N.N. Liu, L.M. Liu, N. Cai, L. Chen, Effect of adenovirus-mediated brain derived neurotrophic factor in early retinal neuropathy of diabetes in rats, Int. J. Ophthalmol. 3 (2) (2010) $145 \mathrm{e} 148$.

[24] H. Fukui, H.T. Wong, L.A. Beyer, B.G. Case, D.L. Swiderski, A. Di Polo, A.F. Ryan, Y. Raphael, BDNF gene therapy induces auditory nerve survival and fiber sprouting in deaf Pou4f3 mutant mice, Sci. Rep. 2 (2012) 838.

[25] T. Nakaizumi, K. Kawamoto, R. Minoda, Y. Raphael, Adenovirus-mediated expression of brainderived neurotrophic factor protects spiral ganglion neurons from ototoxic damage, Audiol. Neurotol. 9 (3) (2004) 135e143.

[26] M.S. Alrashdan, M.A. Sung, Y.K. Kwon, H.J. Chung, S.J. Kim, J.H. Lee, Effects of combining electrical stimulation with BDNF gene transfer on the regeneration of crushed rat sciatic nerve, Acta Neurochir. (Wien) 153 (10) (2011) 2021e2029.

[27] T. Sakamoto, Y. Kawazoe, J.S. Shen, Y. Takeda, Y. Arakawa, J. Ogawa, K. Oyanagi, T. Ohashi, K. Watanabe, K. Inoue, Y. Eto, K. Watabe, Adenoviral gene transfer of GDNF, BDNF and TGF beta 2, but not CNTF, cardiotrophin-1 or IGF1, protects injured adult motoneurons after facial nerve avulsion, J. Neurosci. Res. 72 (1) (2003) 54e64.

[28] C.P. Gomes, A. Varela-Moreira, V. Leiro, C.D. Lopes, P.M. Moreno, M. GomezLazaro, A.P. Pêgo, A high-throughput bioimaging study to assess the impact of chitosan-based nanoparticle degradation on DNA delivery performance, Acta Biomater. 46 (2016) 129e140.

[29] U.S. Food and Drug Administration, Guidance for Industry - Pyrogen and Endotoxins Testing, U.S. Department of Health and Human Services United States, 2012.

[30] G.L. Ellman, Tissue sulfhydryl groups, Arch. Biochem. Biophys. 82 (1) (1959) $70 \mathrm{e} 77$.

[31] H. Oliveira, R. Fernandez, L.R. Pires, M.C. Martins, S. Simoes, M.A. Barbosa, A.P. Pêgo, Targeted gene delivery into peripheral sensorial neurons mediated by self-assembled vectors composed of poly(ethylene imine) and tetanus toxin fragment c, J. Control Release 143 (3) (2010) 350e358.

[32] N.P. Goncalves, H. Oliveira, A.P. Pêgo, M.J. Saraiva, A novel nanoparticle delivery system for in vivo targeting of the sciatic nerve: impact on regeneration, Nanomedicine (Lond.) 7 (8) (2012) 1167 e1180.

[33] M.M. Inserra, D.A. Bloch, D.J. Terris, Functional indices for sciatic, peroneal, and posterior tibial nerve lesions in the mouse, Microsurgery 18 (2) (1998) 119e124.

[34] N.P. Goncalves, M. Teixeira-Coelho, M.J. Saraiva, The inflammatory response to sciatic nerve injury in a familial amyloidotic polyneuropathy mouse model, Exp. Neurol. 257 (2014) $76 \mathrm{e} 87$. 
[35] S. Ferdinandusse, A.W. Zomer, J.C. Komen, C.E. van den Brink, M. Thanos, F.P. Hamers, R.J. Wanders, P.T. van der Saag, B.T. Poll-The, P. Brites, Ataxia with loss of Purkinje cells in a mouse model for Refsum disease, Proc. Natl. Acad. Sci. U. S. A. 105 (46) (2008) 17712e17717.

[36] K.J. Livak, T.D. Schmittgen, Analysis of relative gene expression data using real-time quantitative PCR and the 2(-Delta Delta C(T)) Method, Methods 25 (4) (2001) 402e408.

[37] L. Sarikcioglu, B.M. Demirel, A. Utuk, Walking track analysis: an assessment method for functional recovery after sciatic nerve injury in the rat, Folia Morphol. Warsz. 68 (1) (2009) 1 e7.

[38] M.S. Wang, Y. Wu, D.G. Culver, J.D. Glass, Pathogenesis of axonal degeneration: parallels between Wallerian degeneration and vincristine neuropathy, J. Neuropathol. Exp. Neurol. 59 (7) (2000) 599e606.

[39] J.D. Glass, Wallerian degeneration as a window to peripheral neuropathy, J. Neurol. Sci. 220 (1e2) (2004) 123e124.

[40] T. Gordon, O.A. Sulaiman, A. Ladak, Chapter 24: electrical stimulation for improving nerve regeneration: where do we stand? Int. Rev. Neurobiol. 87 (2009) 433e444.

[41] T. Gordon, N. Tyreman, M.A. Raji, The basis for diminished functional recovery after delayed peripheral nerve repair, J. Neurosci. 31 (14) (2011) 5325e5334.

[42] A. Faroni, S.A. Mobasseri, P.J. Kingham, A.J. Reid, Peripheral nerve regeneration: experimental strategies and future perspectives, Adv. Drug Deliv. Rev. 82e83 (2015) 160e167.

[43] A.E. Dubin, A. Patapoutian, Nociceptors: the sensors of the pain pathway, J. Clin. Investig.Investig. 120 (11) (2010) 3760e3772.

[44] P.B. Toft, K. Fugleholm, H. Schmalbruch, Axonal branching following crush lesions of peripheral nerves of rat, Muscle Nerve 11 (8) (1988) 880e889.

[45] M. Liu, D. Zhang, C. Shao, J. Liu, F. Ding, X. Gu, Expression pattern of myostatin in gastrocnemius muscle of rats after sciatic nerve crush injury, Muscle Nerve 35 (5) (2007) 649e656.

[46] R.H. Wu, P. Wang, L. Yang, Y. Li, Y. Liu, M. Liu, A potential indicator of denervated muscle atrophy: the ratio of myostatin to follistatin in peripheral blood, Genet. Mol. Res. 10 (4) (2011) $3914 \mathrm{e} 3923$.

[47] C. Clow, B.J. Jasmin, Brain-derived neurotrophic factor regulates satellite cell differentiation and skeletal muscle regeneration, Mol. Biol. Cell 21 (13) (2010) 2182e2190.

[48] K. Mousavi, D.J. Parry, B.J. Jasmin, BDNF rescues myosin heavy chain IIB muscle fibers after neonatal nerve injury, Am. J. Physiol. Cell Physiol. 287 (1) (2004) C22eC29.

[49] K. Mousavi, B.J. Jasmin, BDNF is expressed in skeletal muscle satellite cells and inhibits myogenic differentiation, J. Neurosci. 26 (21) (2006) 5739e5749. 


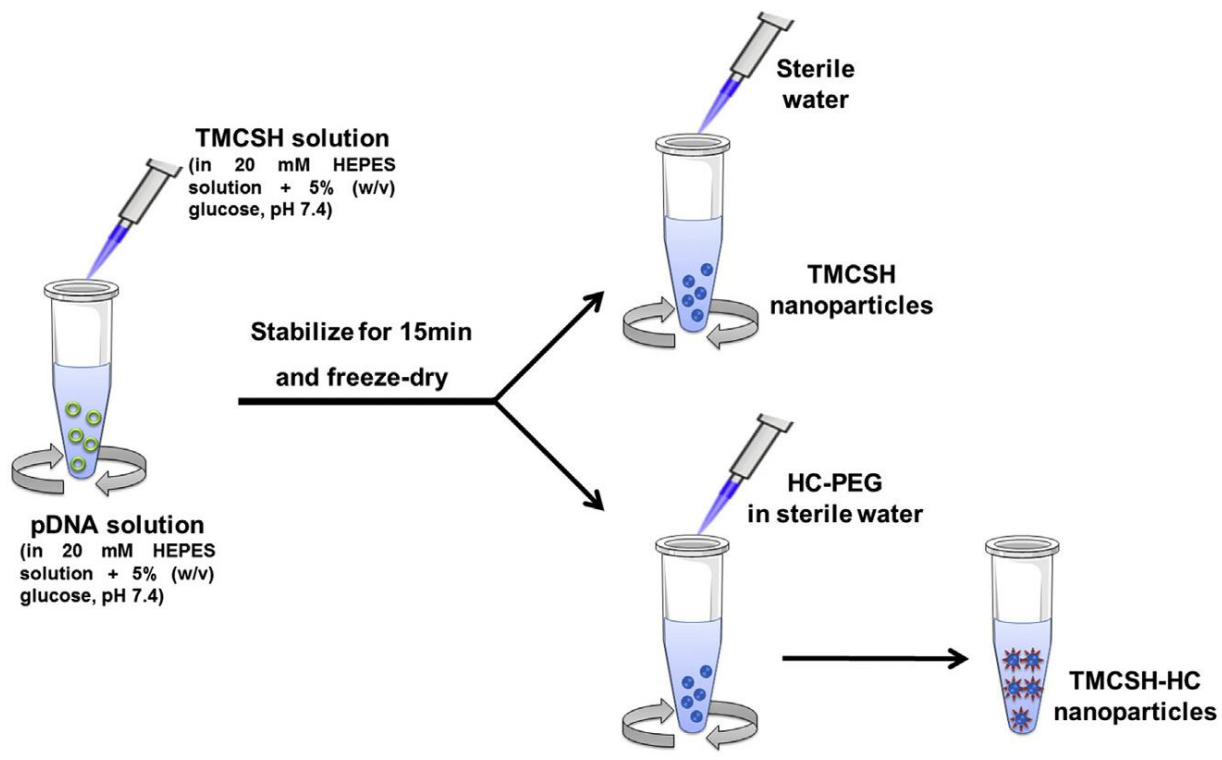

Scheme 1. Schematic illustration of TMCSH-based nanoparticle preparation for in vivo administration. 

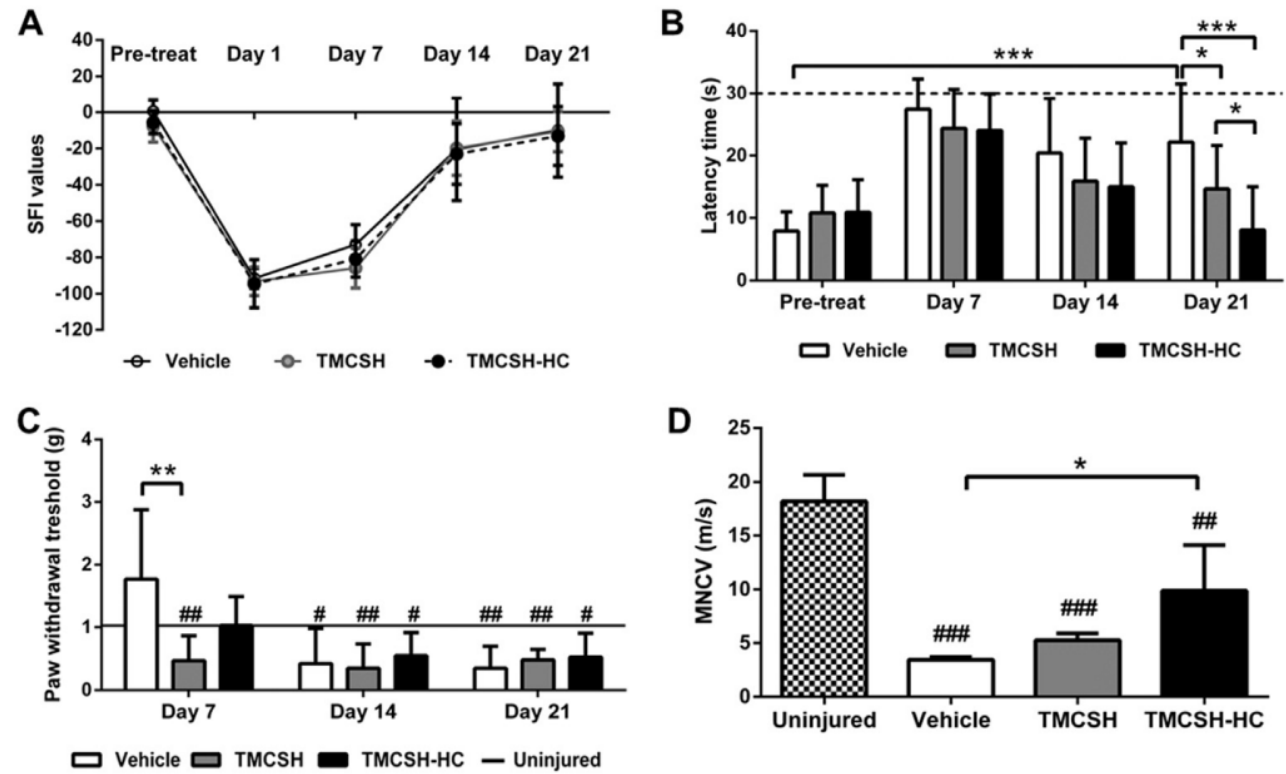

Fig. 1. Sensorimotor functional recovery analyses after sciatic nerve crush injury. (A) Locomotor function recovery assessed by sciatic functional index (SFI) at pre-treatment (Pretreat), 1, 7, 14 and 21 days after nerve crush injury; $n=12$ per group. (B) Thermal nociceptive function recovery measured through the hot plate at pre-treatment (Pre-treat), 7, 14 and 21 days after nerve crush injury; $n=12$ per group. The dashed line represents the $30 \mathrm{~s}$ cut-off time. (C) Mechanical nociceptive function recovery evaluated using the Von Frey filaments at 7, 14 and 21 days after nerve crush injury. Line represents the paw withdrawal threshold in the uninjured paws; $n 1 / 47$ per group. (D) Motor nerve conduction velocities (MNCV) analysis at 21 days after nerve crush injury; $\mathrm{n}=3$ per group. Values represent mean $\pm \mathrm{SD}$. \#\#\# $\mathrm{p}<0.001 \# \# \mathrm{p}<0.01$, \#p $<0.05$ vs uninjured (contralateral). ${ }^{* * *} \mathrm{p}<0.001,{ }^{* *} \mathrm{p}<0.01,{ }^{*} \mathrm{p}<0.05$ in multiple comparisons between treatment groups or time-points of analysis. 
A

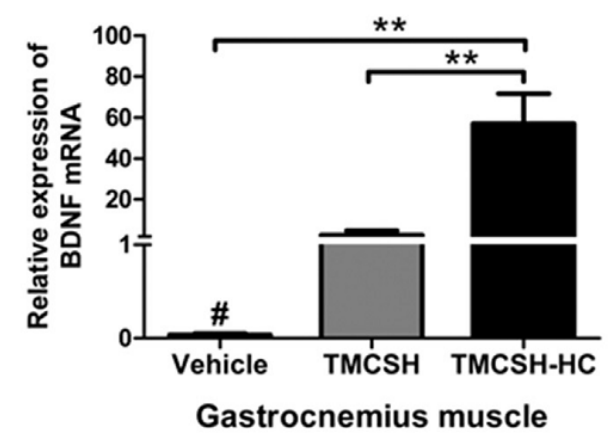

C

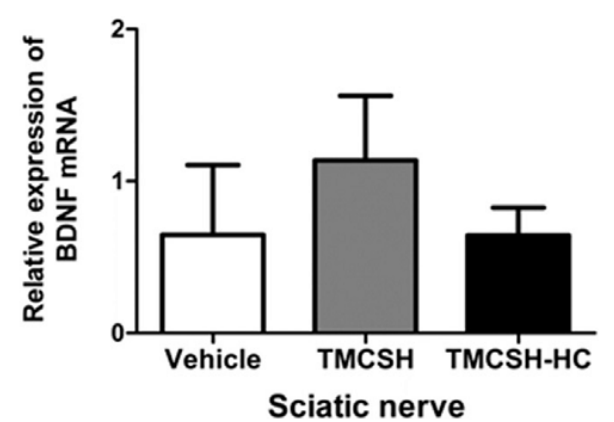

B

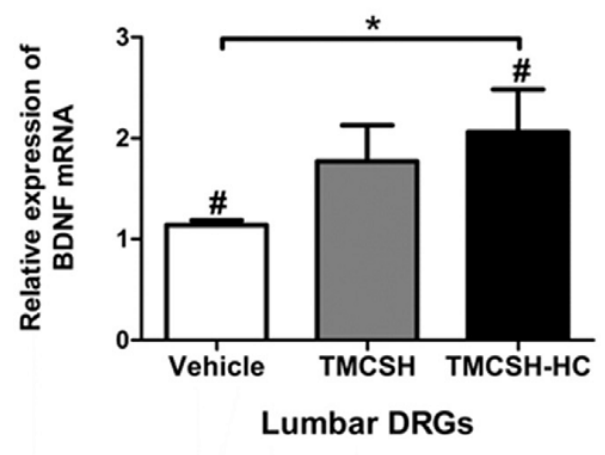

D

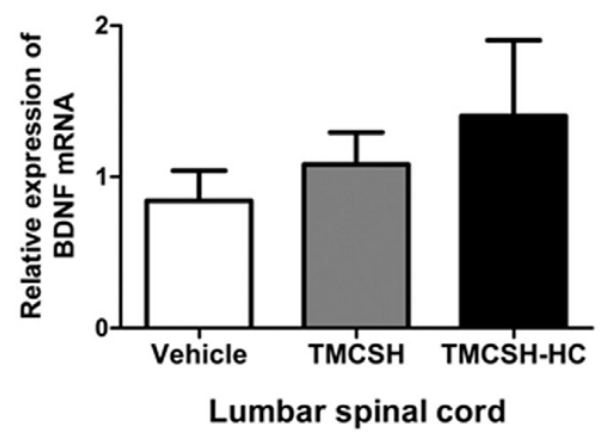

Fig. 2. Relative expression of BDNF mRNA in selected tissues at 21 days after nerve crush injury. BDNF mRNA expression was normalized to GAPDH expression levels and expressed as relative to wild-type animals (uninjured and untreated) in (A) gastrocnemius muscle, (B) lumbar DRGs, (C) sciatic nerve and (D) lumbar spinal cord segments. Values represent mean \pm SD; $n=3$ per group. ${ }^{*} \mathrm{p}<0.05$ vs wild-type animals. ${ }^{* *} \mathrm{p}<0.01,{ }^{*} \mathrm{p}<0.05$ in multiple comparisons between treatment groups. 


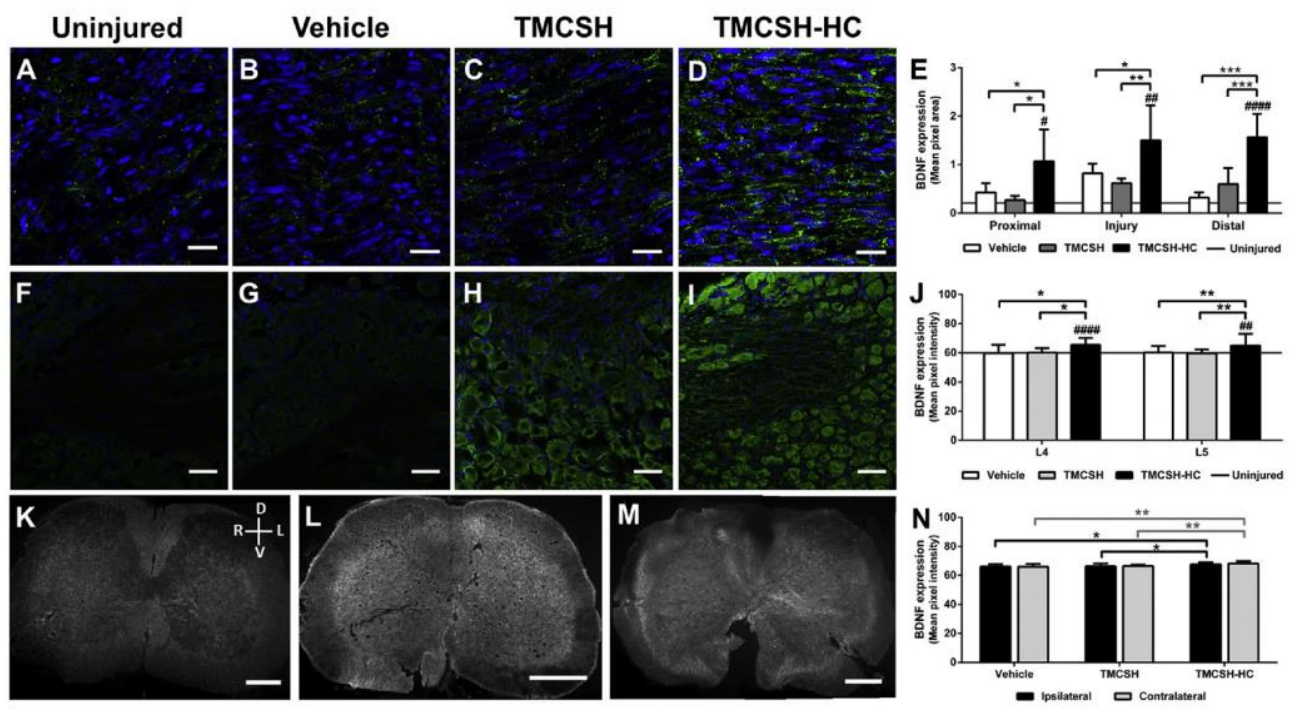

Fig. 3. BDNF protein expression profile. BDNF protein was detected by immunohistochemistry in the sciatic nerve, lumbar DRGs and lumbar spinal cord, at 21 days after nerve crush injury. (A-D) Representative images of sciatic nerve distal segments immunostained for BDNF (green) from animals in the (A) uninjured (contralateral), (B) vehicle, (C) TMCSH and (D) TMCSH-HC groups; Nuclei stained with DAPI (blue). Scale bars represent $25 \mu \mathrm{m}$. (E) Semi-quantification of BDNF protein expression in the proximal, injury and distal portions of sciatic nerves. Line represents the BDNF expression levels in uninjured nerves (contralateral). Values represent the mean area occupied by pixels corresponding to the fluorescent signal normalized in relation to the total area (mean \pm SD); $\mathrm{n}=5$ per group. \#\#\#\# $\mathrm{p}<0.0001$, ${ }^{\# \# p ~}<0.01$, ${ }^{\#} \mathrm{p}<0.05$ vs uninjured. ${ }^{* * *} \mathrm{p}<0.001,{ }^{* *} \mathrm{p}<$ $0.01,{ }^{*} p<0.05$ in multiple comparisons between treatment groups. (FeI) Representative images of lumbar DRG immunostained for BDNF (green) from animals in the (F) uninjured (contralateral) (G) vehicle, (H) TMCSH and (I) TMCSH-HC group; Nuclei stained with DAPI (blue). Scale bars represent $50 \mathrm{~mm}$. (J) Semi-quantification of BDNF protein expression in the L4 and L5 DRGs. Line represents the BDNF expression levels in contralateral DRGs. Values represent the mean pixel intensity levels $\pm \mathrm{SD} ; \mathrm{n}=5$ per group. $\# \# \# \mathrm{p}<0.0001$, ${ }^{\# \#} \mathrm{p}<0.01$ vs contralateral DRGs ${ }^{* *} \mathrm{p}<0.01$, $* \mathrm{p}<0.05$ in multiple comparisons between treatment groups. (KeM) Representative grayscale images of lumbar spinal cord hemisection immunostained for BDNF from the (K) vehicle, (L) TMCSH and (M) TMCSH-HC group; D: dorsal, V: ventral, R: right, L: left; Scale bars represent 500 $\mathrm{mm}$. (N) Semi-quantification of BDNF protein expression in the lumbar spinal cord. Values represent the mean pixel intensity levels \pm SD; $n=5$ per group. ${ }^{* *} \mathrm{p}<0.01,{ }^{*} \mathrm{p}<0.05$ in multiple comparisons between treatment groups. (For interpretation of the references to colour in this figure legend, the reader is referred to the web version of this article.) 

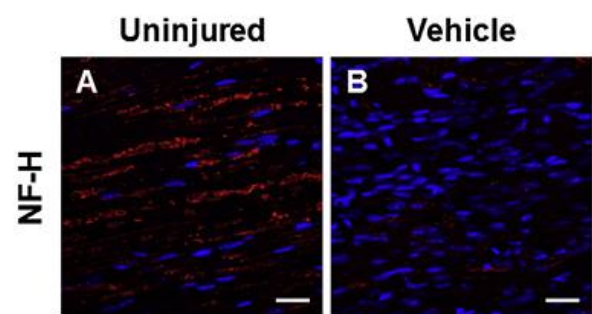

TMCSH

TMCSH-HC
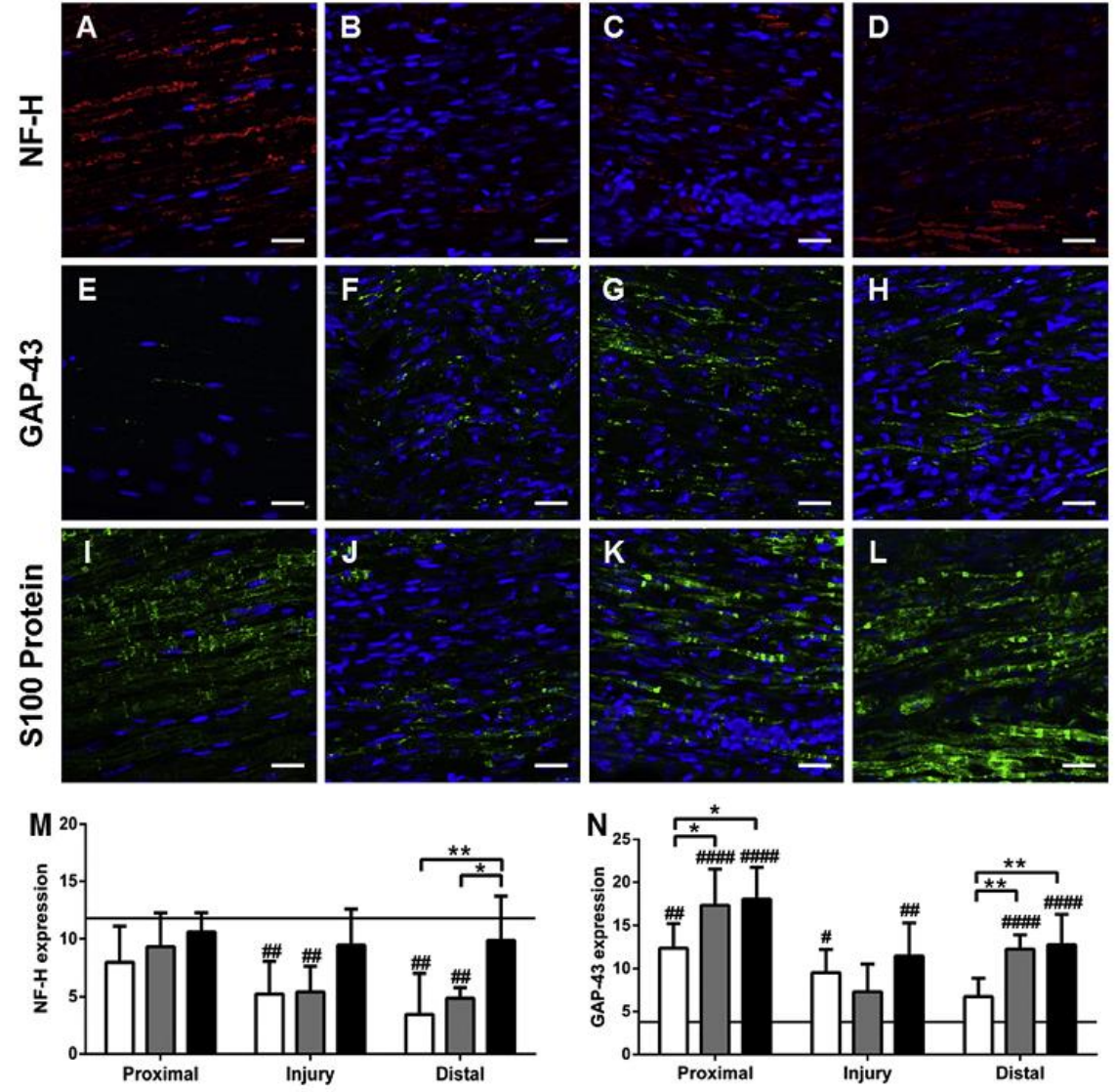

Vehicle $\square$ тмCSH TMCSH.HC—Uninjured

$\square$ Vehicle $\square$ тMCSH $\square$ TMCSH-HC — Uninjured

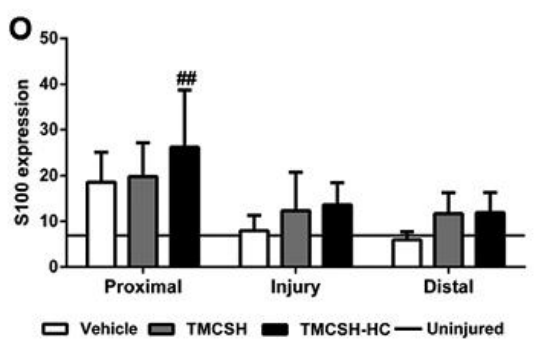

Fig. 4. Histological analysis of injured sciatic nerves at 21 days after nerve crush injury. (A-D) Immunohistochemistry representative images of sciatic nerve sections for the detection of NF-H (red) from (A) uninjured nerves, (B) vehicle group, (C) TMCSH group and (D) TMCSH-HC group. (EeH) Immunohistochemistry representative images of sciatic nerve sections for the detection of growth associated protein GAP-43 (green) from (E) uninjured nerves (contralateral), (F) vehicle group, (G) TMCSH group and (H) TMCSH-HC group. (I-L) Immunohistochemistry representative images of sciatic nerve sections for the detection of S100 protein (green) from (I) uninjured nerves, (J) vehicle group, (K) TMCSH group and (L) TMCSH-HC group. Nuclei stained with DAPI (blue). Scale bars represent $25 \mu \mathrm{m}$ (M-O) Semi-quantification of NF-H (M), GAP-43 (N) and S100 protein expression $(0)$ in the sciatic nerve sections. Line represents the respective marker expression levels in the uninjured nerves (contralateral). Values represent mean $\pm \mathrm{SD} ; \mathrm{n}=5$ per group. \#\#\# $\mathrm{p}<$ $0.0001,{ }^{\# \#} \mathrm{p}<0.01,{ }^{\#} \mathrm{p}<0.05$ vs uninjured nerves. ${ }^{* *} \mathrm{p}<0.01,{ }^{*} \mathrm{p}<0.05$ in multiple comparisons between treatment groups. (For interpretation of the references to colour in this figure legend, the reader is referred to the web version of this article.) 

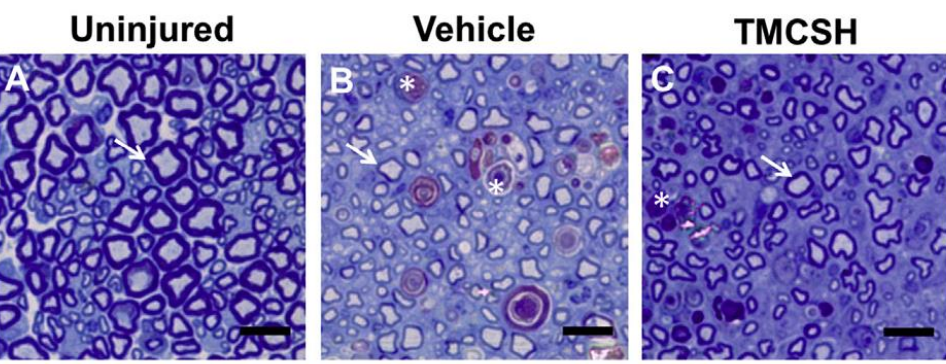

\section{TMCSH-HC}
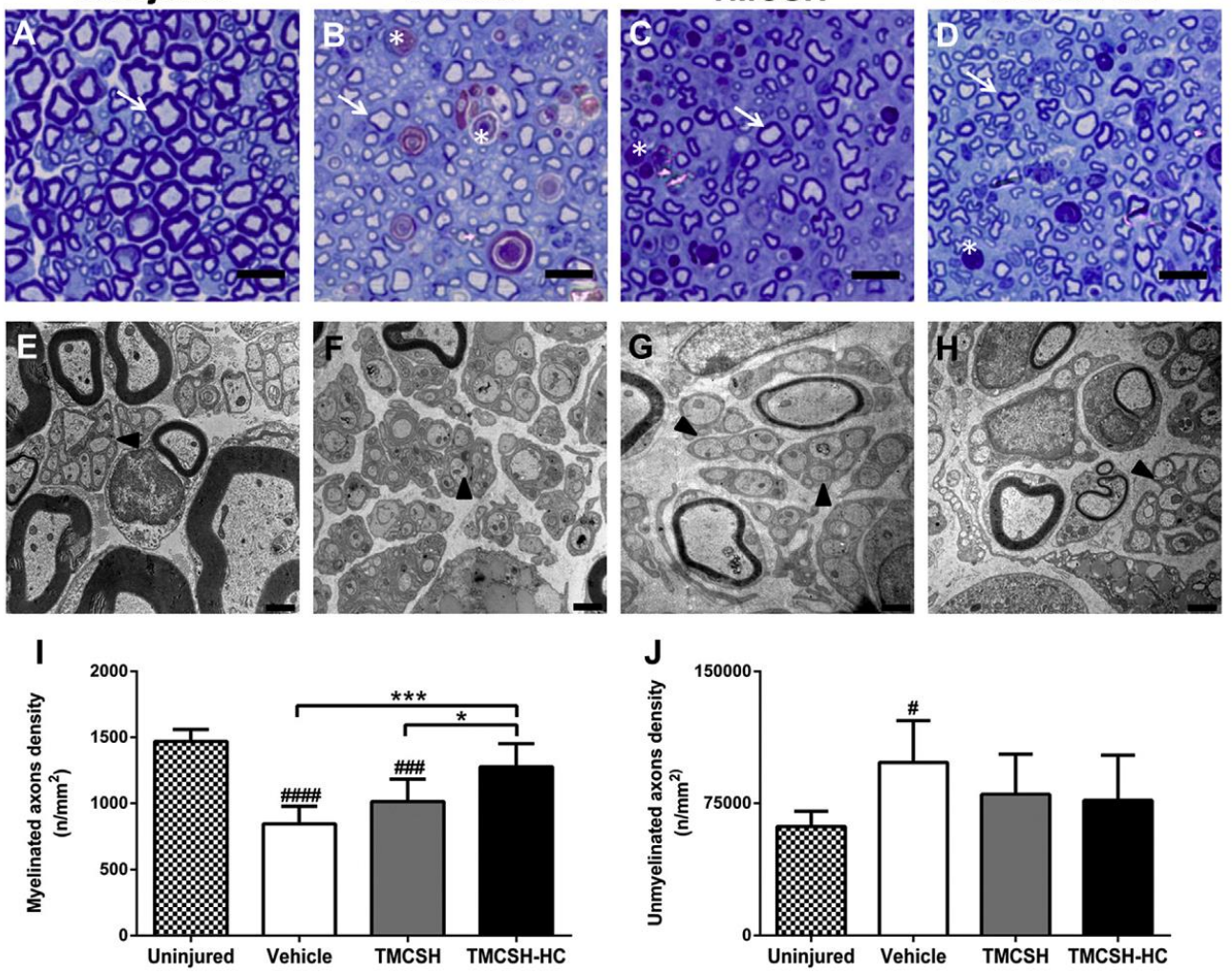

Fig. 5. Morphometric analysis of distal portions of sciatic nerves at 21 days after nerve crush injury. (A-D) Representative images of semi-thin transverse sciatic nerve sections from (A) uninjured nerves (contralateral), (B) vehicle group, (C) TMCSH group and (D) TMCSH-HC group. Arrows indicate myelinated axons; ${ }^{*}$ depicts degenerating axons. Scale bar represents $25 \mu \mathrm{m}$. (E-H) Representative images of ultra-thin transverse sciatic nerve sections from (E) uninjured nerves (contralateral), (F) vehicle group, (G) TMCSH group and (H) TMCSH-HC group. Arrowheads show Remak bundles. Scale bar represents $1 \mu \mathrm{m}$. (I) Density of myelinated axons expressed as number of myelinated axons per square millimeter $\left(\mathrm{n} / \mathrm{mm}^{2}\right)$. (J) Density of unmyelinated axons expressed as number of unmyelinated axons per square millimeter $(\mathrm{n} / \mathrm{mm} 2)$. Values represent mean $\pm \mathrm{SD} ; \mathrm{n}$ $=5$ per group. $\# \# \#$ \# $<0.0001, \# \#$, $<0.001$ vs uninjured nerves. ${ }^{* * *}$ p $<0.001,{ }^{*} p<0.05$ in multiple comparisons between treatment groups. 


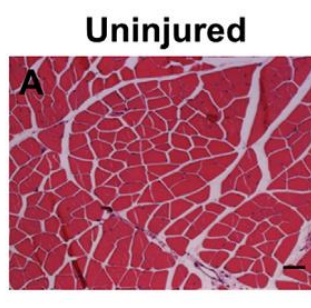

E

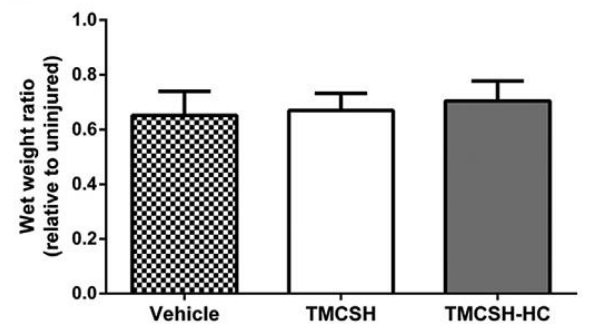

G
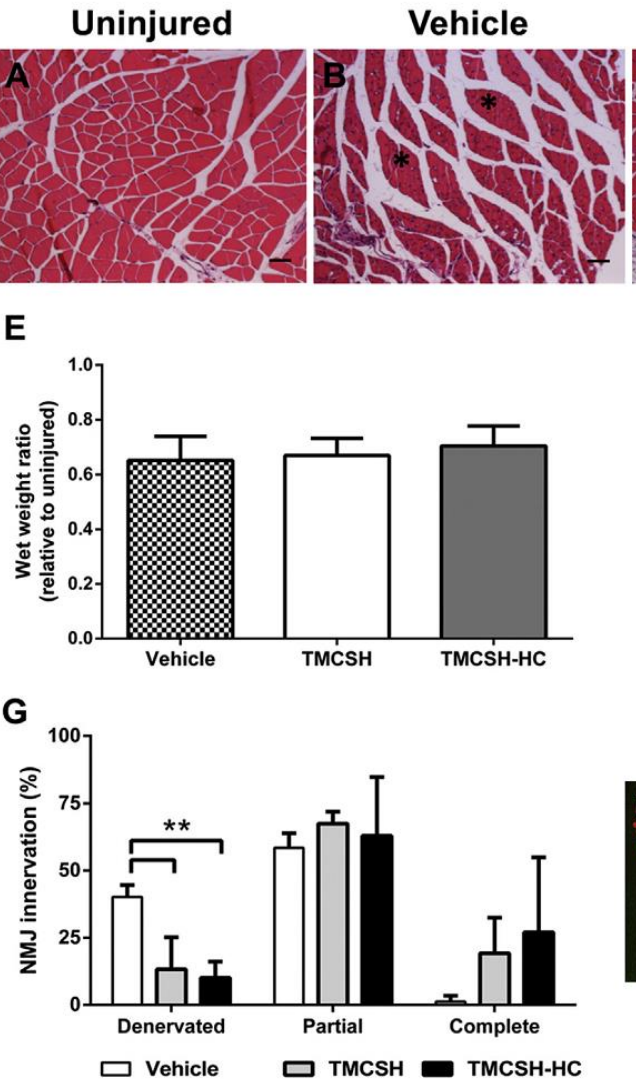

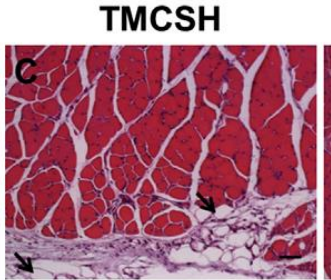

TMCSH-HC

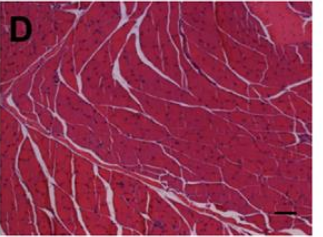

$\mathbf{F}$
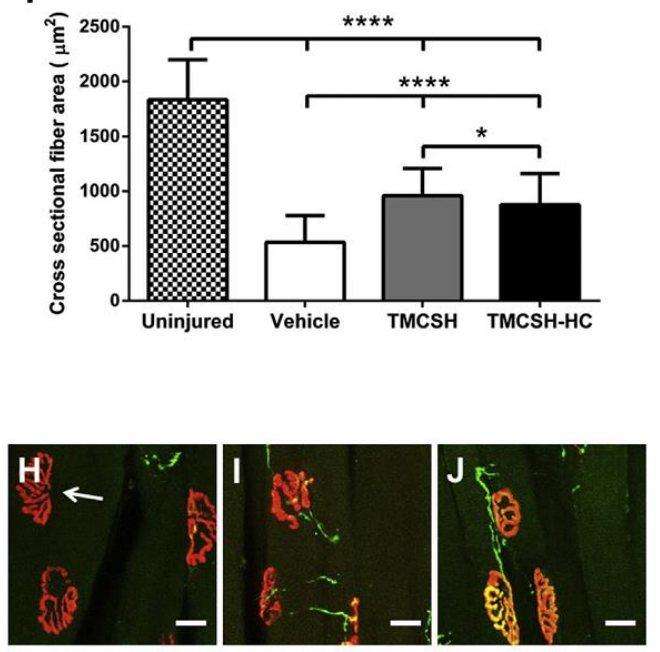

Fig. 6. Morphometric analysis of gastrocnemius muscles at 21 days after nerve crush injury. Representative images of gastrocnemius muscles from (A) uninjured (contralateral), (B) vehicle group, (C) TMCSH group and (D) TMCSH-HC group. * Depicts groups of atrophic muscle fibers; Arrows indicate the presence of adipocytes. Scale bar $50 \mu \mathrm{m}$. (contralateral) (E) Gastrocnemius muscle wet weight represented as the ratio between muscle wet weight in the injured limb relative to the uninjured muscle wet weight (uninjured limb); $\mathrm{n}=12$ per group. (F) Cross sectional area $(\mu \mathrm{m} 2)$ of gastrocnemius muscle fibers; $n=3$ per group. (G) Quantification of fraction of NMJ innervation from at least 50 NMJs; $n=3$ per group. 100\% represents a tissue where all NMJ are completely innervated (uninjured). (H-J) Representative images of denervated (H, arrow), partially innervated (I) and completely innervated NMJs (J) from gastrocnemius muscle using the NF200 marker (green) and the postsynaptic marker $\alpha$-bungarotoxin (red) at 21 days after injury. Scale bar represents $20 \mu \mathrm{m}$. Values represent mean $\pm \mathrm{SD}$. ${ }^{* * * *} \mathrm{p}<0.0001,{ }^{* *} \mathrm{p}<0.01,{ }^{*} \mathrm{p}<0.05$. (For interpretation of the references to colour in this figure legend, the reader is referred to the web version of this article.) 
Table 1 - Physicochemical characterization of TMCSH-based nanoparticles at an N/P ratio (moles of quaternized amine groups $(\mathrm{N})$ to moles of DNA phosphate groups (P)) of 2. Nanoparticles were characterized in terms of size, polydispersity index (Pdi) and zeta potential in $20 \mathrm{mM}$ HEPES buffered saline solution containing $5 \%(\mathrm{w} / \mathrm{v})$ glucose, $\mathrm{pH} 7.4$. All values are representative of 3 independent experiments and are expressed as mean \pm SD.* Statistically different from TMCSH formulation $(\mathrm{p}<0.05)$.

\begin{tabular}{llll}
\hline Formulations & Size $(\mathrm{nm})$ & Pdi & Zeta potential $(\mathrm{mV})$ \\
\hline TMCSH & $270.8 \pm 5.7$ & $0.16 \pm 0.02$ & $+19.1 \pm 0.5$ \\
TMCSH-HC & $262.8 \pm 1.6$ & $0.17 \pm 0.01$ & $+17.2 \pm 0.9^{*}$ \\
\hline
\end{tabular}

